\title{
On the propagation and multiple reflections of a blast wave travelling through a dusty gas in a closed box
}

\author{
Marcello Lappa ${ }^{*}$, Dimitris Drikakis, Ioannis Kokkinakis \\ Faculty of Engineering, University of Strathclyde, James Weir Building, 75 Montrose Street, \\ Glasgow, G1 1XJ, UK \\ *Corresponding author email: marcello.lappa@strath.ac.uk
}

\begin{abstract}
This paper concerns the propagation of shock waves in an enclosure filled with dusty gas. The main motivation for this problem is to probe the effect on such dynamics of solid particles dispersed in the fluid medium. This subject, which has attracted so much attention over recent years given its important implications in the study of the structural stability of systems exposed to highenergy internal detonations, is approached here in the framework of a hybrid numerical two-way coupled Eulerian-Lagrangian methodology. In particular, insights are sought by considering a relatively simple archetypal setting corresponding to a shock wave originating from a small spherical region initialized on the basis of available analytic solutions. The response of the system is explored numerically with respect to several parameters, including the blast intensity (via the related value of the initial shock Mach number), the solid mass fraction (mass load), and the particle size (Stokes number). Results are presented in terms of pressure-load diagrams. Beyond practical applications, it is shown that a kaleidoscope of fascinating patterns is produced by the "triadic" relationships among multiple shock reflections events and particle-fluid and particle-wall interaction dynamics. These would be of great interest to researchers and scientists interested in fundamental problems relating to the general theory of pattern formation in complex nonlinear multiphase systems.
\end{abstract}

\section{Introduction}

Two-phase flow phenomena present an important field of study for a variety of systems. The breadth of applicability of such studies is large, ranging from several practical applications to natural processes and even problems of astrophysical interest. ${ }^{1,2}$

Related examples for the case of highly compressible flows include sprays in high-speed jet engines (air-breathing propulsion systems in which liquid-fuel droplets are typically dispersed through a gaseous environment ${ }^{3}$ ) and a number of technological processes for the production of powder materials. $^{4}$

Compressibility effects (and shock waves) are also relevant to the behavior of exhaust plumes of rocket motors propelled by solid fuels. Recent studies along these lines have been undertaken for the development of new propulsion systems such as the so-called pulsed detonation engine. ${ }^{5}$ These problems also underpin research in catalytic processes, mineral processing, and nuclear reprocessing. In such contexts, of particular interest are the dynamics of shock waves that either are initiated within a dusty gas or propagate into a gas-particle mixture. Typically, such information is needed to understand the dynamics associated with detonations and explosions in confined areas (e.g., shock waves that occur while conveying particles pneumatically in chemical plants, grain 
mills, and mine galleries ${ }^{6,7}$ ). Other related applications can be found in the field of civil engineering, where a need has arisen recently for new safety criteria in the structural design of buildings (see, for example, the review by $D$ hakal ${ }^{8}$ ). Similar motivations are behind studies concerned with the simulation of pyroclastic flows resulting from explosive volcanic activity. ${ }^{9}$ Because of the hazardous nature of such events, an accurate assessment of the areas invaded by the flows and a quantitative estimate of their destructive actions (in terms of such measures as temperature and dynamic pressure) is of great importance for civil protection purposes. ${ }^{10}$

Most previous studies have focused essentially on the "shock-tube" problem, given the undeniable advantages offered by this specific configuration, which can be regarded as very versatile shortduration facilities ${ }^{11-19}$ Sommerfeld ${ }^{6}$ was the first to attack the problem experimentally by studying directly the attenuation of a shock as it propagates into a gas-particle mixture. A number of tests were conducted at different initial shock speeds $M_{s}$ and particle loadings. These experimental tests revealed the incredible multiscale nature of such a seemingly innocuous problem (see also the subsequent experimental study by Boiko et al. ${ }^{4}$ ).

Over subsequent years, paralleling such new conceptual understanding was the development of powerful numerical codes. Among such numerical studies (essentially relying on the earlier experimental results by Sommerfeld ${ }^{6}$ for model validation), it is worth citing Olim et al. ${ }^{20}$ and Aizik et al., ${ }^{21}$ who expressly used the results of their calculations to devise a general analytic law for predicting the attenuation of the shock wave (a kind of exponential decay curve) as a function of particle loading and diameter changes. The interplay between numerical simulation and analytic arguments was particularly fruitful (the main drawback of such laws, however, is they cannot be used to predict the effective "pressure load" exerted by a shock wave on solid boundaries).

More recent and modern attempts (in terms of numerical strategies used to solve the problem) are due to Sivier et al., ${ }^{3}$ Loth et al., ${ }^{22}$ and Chang and Kailasanath, ${ }^{5}$ for example. Such analyses provide important additional and more precise details on the influence of a number of parameters. Scarce attention, however, was paid to the interplay between shock waves and solid walls. In addition, the considered dynamics were still essentially one-dimensional.

In the present work, we concentrate expressly on the fundamental interaction of a shock wave originating from a localized region inside a closed cavity with the related solid boundary, including the multiple shock reflections that take place when the shock wave bounces off the walls, then off the core region at small density, and then again toward the walls. These flows hide a still-not-fullyunderstood competition of complex and diverse physical mechanisms that operate on very different spatial and temporal scales. The essentially spherical nature of the shock wave during its initial stages of evolution and the presence of straight walls that break such a symmetry make typical earlier one-dimensional approaches inapplicable to these processes.

Apart from a couple of pioneering studies ${ }^{23,24}$ that were limited to "pure" gas (no dispersed solid mass), this important category of problems has not received the attention it deserves. Additional experimental and (especially) computational studies are needed to provide detailed analysis of such flow fields and to allow increased understanding for improved engineering design. 


\section{Mathematical and Numerical Model}

The influence of dispersed mass on such phenomena is still a matter of debate. This field continues to burgeon and yield surprises. This is due in large part to the difficulties of setting up in laboratory tests a suitable well-controllable flow that would also allow a wide variety of dynamical regimes to be explored. As a valid alternative to past optimization strategies based on expensive trial-and-error experiments, recent progress in numerical techniques and computing hardware has provided a powerful framework for sophisticated computer-aided design, modeling, and simulation.

A full understanding of such a problem requires it particularly to be modeled in a consistent way on many levels simultaneously. The first of these levels is to represent the highly compressible flow resulting from the propagation of a shock wave. The related mathematical formalism must be sufficiently general to allow a wide class of regimes to be considered and, at the same time, simple enough not to increase excessively the complexity of the resulting governing equations. The latter, in turn, must allow the flow in the gas to be simulated as completely as possible.

The second level of such a modeling hierarchy is to simulate the motion of the dispersed phase itself. Physical and mathematical modeling of these processes requires sophisticated understanding of the relaxation processes behind the shock-wave front at temperatures and pressures that are characteristic of the considered problem. The presence of an inert dispersed phase will act to attenuate, or decelerate, the passing shock through momentum and energy extraction.

More specifically, as a shock wave propagates into a gas-particle mixture, the gas velocity increases instantaneously across the shock and the shock velocity is decreased by the presence of dispersed solid mass. In contrast, the particle velocity only slowly approaches the post-shock gas velocity because of the finite inertia of the particles.

When solving the compressible two-phase equations, the continuum gas dynamics are usually best represented by an Eulerian description, that is, the gas characteristics are calculated at fixed locations (grid points) in the flow. However, as the particles may be relatively sparse in the flow field, they can be modeled by either an Eulerian description (in the same way as the gas flow, i.e. by assuming that they behave essentially as a continuous phase) or a Lagrangian description (in which individual particle groups are monitored and tracked in the flow). Both descriptions have enjoyed widespread use in past studies relating to the aforementioned shock-tube problem. ${ }^{25}$

Each method, of course, has its own advantages and disadvantages. Eulerian methods allow particle diffusion to be incorporated directly into the model via the addition of some "production terms" to the momentum and energy equations. As illustrated by many authors (see, e.g., Marble ${ }^{26}$ or Sommerfeld et al. ${ }^{6}$, however, the adoption of an "equivalent gas model" becomes necessary for the gas-particle mixture. The properties of such a mixture are typically determined using relationships which "combine" the properties of the pure gas and of the dispersed phase. This has an impact on the equation of state for the mixture and the $\gamma$ parameter.

The above additional modeling is not required when a hybrid Lagrangian-Eulerian approach is used, provided the coupling between the fluid and solid phase is of a two-way nature (as we will show in 
detail in Sect. IIC, such an issue is generally implicitly resolved by the model, in other words there is no need to consider modified gas properties or gas state equations). For this reason (and also because Lagrangian methods typically require less modeling effort to account for particles rebounding off boundary surfaces), in the present work, we resort to the second approach with the express intention of developing an algorithm capable of targeting the broadest range of situations.

\section{A. Fluid governing equations}

We consider the inviscid two-dimensional Euler equations, which, for a compressible fluid, can be cast in condensed (matrix) form as

$$
\frac{\partial \underline{q}}{\partial t}+\frac{\partial \underline{F}}{\partial x}+\frac{\partial \underline{G}}{\partial y}=\underline{S}_{p} \text {, }
$$

where the vector $q$ contains the conservative variables $q=\left[\rho_{g}, \rho_{g} u, \rho_{g} v, \rho_{g} E\right], \rho_{g}$ being the density of the gas, $u$ and $v$ its velocity components along the $x$ and $y$ directions, respectively, and $E$ the total energy per unit mass. The related inviscid fluxes, in turn, read:

$$
\underline{F}=\left[\begin{array}{c}
\rho_{g} u \\
\rho_{g} u^{2}+p \\
\rho_{g} u v \\
\rho_{g} u\left(E+\frac{p}{\rho_{g}}\right)
\end{array}\right] \quad \underline{G}=\left[\begin{array}{c}
\rho_{g} v \\
\rho_{g} v u \\
\rho_{g} v^{2}+p \\
\rho_{g} v\left(E+\frac{p}{\rho_{g}}\right)
\end{array}\right]
$$

where $E=C_{v} T+\frac{1}{2}\left(u^{2}+v^{2}\right)$ and $p=\rho_{g} R T$ (the gas state equation, $R$ being the gas constant). The additional vector term $\underline{S}_{p}$ in the right-hand side of Eq. (1) is required to properly couple the liquid and solid phases, as we shall further detail in Sect. IIC.

\section{B. Particle tracking equations}

Given the considered topic, the present problem may be regarded as a typical example of situations in which one witnesses interplay between large-scale (shock-wave propagation inside the considered enclosure) and small-scale entities and processes (particles and their motion under the influence of the large-scale flow), which, in general, require a multiscale (and multiphysics) approach. Accordingly, two models have to be defined to close the problem properly from a mathematical point of view: one referring to the nature of a large-scale flow, and the other dealing with issues strictly related to the displacement of particles in time and associated mechanisms. In other words, this means that some model diversification must be introduced on the basis of the 
desired length scale, i.e. according to the level of detail required by the considered phenomena, these being "bulk flow" or "particle motion."

We have covered the former case in Sect. IIA in the framework of an Eulerian representation of the flow field based on inviscid equations (viscous effects neglected). The latter requires a treatment that depends on the specific situation involved and especially on the "nature" of the dispersed phase. In the present case, the density $\rho_{s}$ of the particles is much larger than the density $\rho_{g}$ of the surrounding gas $\left(\xi=\rho_{s} / \rho_{g}>>1\right.$, which means that inertia of the former is significant). Moreover, the particles are much smaller than the characteristic length scale of the considered flow, which leads to the obvious conclusion that viscous effects should be adequately taken into account at the particle length scale.

Given such arguments, and taking into account that the dispersed phase is constituted of small particles that undergo neither dimensional changes nor shape modification (they are assumed to be undeformable), as anticipated in Sect. II, we resort to a "hybrid" formulation. This is an Eulerian approach to determine the large-scale flow (Eqs. (1) and (2)) and a properly coupled Lagrangian method to capture particle motion.

Accordingly, we treat particles as isolated, microscopic quantities compared with field variables, and track the motion of the former by using an appropriate equation expressing the balance between particle inertia and viscous drag forces. Such an equation (see, e.g., Chang and Kailasanath ${ }^{5}$ ) can be cast in compact vector form as

$\rho_{s} \frac{d \underline{V}_{p}}{d t}=\frac{9}{2} \frac{\mu}{R_{p}^{2}} f\left(\operatorname{Re}_{p}\right)\left(\underline{V}-\underline{V}_{p}\right)$,

where $\underline{V}_{p}=\left[u_{p}, v_{p}\right]$ is the particle velocity and $\underline{V}=[u, v]$ is the local gas velocity. Moreover, $R_{p}$ is the particle radius (we assume particles to be perfectly spherical) and $R e_{p}$ is the related instantaneous Reynolds number, defined as

$\operatorname{Re}_{p}=\frac{2 R_{p} \rho_{g}\left|\underline{V}-\underline{V}_{p}\right|}{\mu}$.

The additional term $f\left(R e_{p}\right)$ appearing in Eq. (3) is a corrective factor required to account for the departure of the drag from the classical Stokes law: $:^{5,27}$

$f\left(\operatorname{Re}_{p}\right)=1+0.15 \operatorname{Re}_{p}^{0.687}$

The gas dynamic viscosity $\mu$ is computed according to Sutherland's law on the basis of the local (at particle position) temperature:

$\mu_{\text {Suth }}=\mu_{o}\left(\frac{T}{T_{o}}\right)^{3 / 2} \frac{T_{o}+S}{T+S}$, 
where $\mu_{o}$ is a reference viscosity (in the same units as $\mu$ ) at a reference temperature $T_{o}$. The gas velocity $\underline{V}$ appearing in Eqs. (3) and (4) must be "reconstructed" at each particle location from the surrounding grid locations, which requires a proper interpolation scheme for problem closure (we will come back to this concept, which requires some additional considerations, in Sect. IIC)

In addition to the previous mathematical modeling, as another (but not independent) characteristic parameter, we introduce the particle Stokes number, defined as the ratio between the particle relaxation time $(\tau)$ :

$$
\tau=\frac{2}{9} \frac{R_{p}^{2}}{v_{o}}
$$

and the characteristic viscous time scale of carrier flow:

$$
\tau_{\text {flow }}=\frac{L^{2}}{v_{o}}
$$

where $L$ is the characteristic size of the considered geometrical domain ${ }^{28}$, and $v$ the fluid kinematic viscosity). The resulting non-dimensional ratio reads

$$
S t=\frac{2}{9}\left(\frac{R_{p}}{L}\right)^{2},
$$

which must be $<1$ to make Eq. (3) valid (a prerequisite for the applicability of Eq. (3) is that $R_{p}<<L$ ).

\section{Two-way model}

In so-called "one-way" coupled systems, the particle mass loading is assumed to be sufficiently small so that any effects of the dispersed phase on the gas phase can be ignored. In other words, the local gas-phase velocity is assumed to have a direct influence on particle motion, but not vice versa. However, in the cases examined here, the particle mass loading is high enough that return effects cannot be considered negligible; a two-way coupled approach must be invoked and implemented accordingly. This is accomplished via the presence of the interphase coupling term $S_{p}$ in Eq. (1), which we have formally introduced in Sect. IIIA. To account for the "return" effects from the dispersed particles to the gas phase, this term must be properly computed for each grid cell. The related implementation requires an algorithm to determine the number of particles present at a given instant in any computational cell of the domain. By denoting this number as $n_{i j}$ ( $i$ and $j$ being the representative indexes of the $x$ and $y$ directions, respectively), this interphase term can be computed as $\underline{S}_{p}=\left[S_{\rho}, S_{\rho u}, S_{\rho v}, S_{\rho E}\right]$, where 


$$
\begin{aligned}
& S_{\rho}=0 \\
& S_{\rho u}=-\sum_{k=1}^{n_{i j}} \frac{m_{p k}}{\delta \Omega_{i j}} \frac{d u_{p k}}{d t}, \\
& S_{\rho v}=-\sum_{k=1}^{n_{i j}} \frac{m_{p k}}{\delta \Omega_{i j}} \frac{d v_{p k}}{d t}, \\
& S_{\rho E}=-\sum_{k=1}^{n_{i j}} \frac{m_{p k}}{\delta \Omega_{i j}}\left[u_{p k}\left(\frac{d u_{p k}}{d t}\right)+v_{p k}\left(\frac{d v_{p k}}{d t}\right)\right] .
\end{aligned}
$$

The first component is zero because we do not consider mass exchange between the two phases. The other components can be used to model the exchange of momentum and energy between the gas and the dispersed solid material. The sign minus in front of such terms indicates that an acceleration of particles $\left(\mathrm{d} \underline{V}_{p} / \mathrm{d} t>0\right)$ would be reflected in a corresponding deceleration of the gas $(\mathrm{d} \underline{V} / \mathrm{d} t<0)$, and vice versa. The quantities $m_{p}$ and $\delta \Omega_{i j}$ are the mass of the generic particle and the volume of the computational cell containing it, respectively: $m_{p}=\rho_{s} \frac{4}{3} \pi R_{p}^{3}$ and $\delta \Omega_{i j}=\Delta x \Delta y 2 R_{p}$.

Following Sivier et al. ${ }^{3}$ and Loth et al. ${ }^{22}$, we assume that the gas pressure is not influenced by the particle volumes because of their negligible overall volume fraction $\left(\mathrm{O}\left(10^{-3}\right)\right.$ for the conditions considered in the present work).

Moreover, we do not model here the heat exchange between particles and the surrounding fluid, which would require the solution of an additional Lagrangian equation and the addition of another source term to eq. (10d). Though we acknowledge that the consideration of such a heat-exchange term would further increase the physical consistency of the present approach, we expressly choice not to consider it because its presence would introduce significant additional mathematical complexity, without having however a significant quantitative impact on the results (its influence being expected to be relatively scarce for mass loads of unit order such as those considered here). In the following, we denote by $\phi$ the ratio between the volume of the generic particle and the volume of the computational cell, i.e., $\phi=\frac{2}{3} \pi R_{p}^{2} / \Delta x \Delta x$. An intrinsic requirement of such an approach is that $\phi<<1$ so that particles can be treated as microscopic quantities compared with the size of a typical mesh cell. Notably, such a condition puts a constraint on the maximum number of grid points that can be used to treat the problem, or alternatively on the maximum tolerable size of the particles for a given mesh spacing. Another constraint is the maximum number of particles that can be handled effectively (the upper limit being set essentially by the characteristics of the computer used for the numerical simulations, i.e., computational speed and available memory). Following widespread practice in the existing literature (e.g., Chang and Kailasanath ${ }^{5}$ ), in order to achieve high mass loadings without the necessary computational expense of tracking every particle in the system, we resort to a virtual particle method. This means each particle in the simulation acts as a marker or carrier for a group of virtual particles, the center of mass of which is located at the 
simulation particle position. It is assumed that each of the virtual particles has the size and mass of the simulation particle. However, the velocity and location of the former are not explicitly calculated because they are assumed to move with their associated carrier particle. Nevertheless, the momentum and energy from the virtual particles are included in the coupling-feedback source terms and are assumed to be located at the same position as the carrier particle.

By denoting the mass load (namely, the ratio of the overall solid mass present in the computational domain to the total mass of gas) by $\eta$ and the number of particles effectively tracked by the algorithm (limited from above by the aforementioned constraints on computer speed and memory) by $N_{\text {track}}$, the number of virtual particles per each effectively tracked particle can be computed according to the following relationship:

$$
N_{\text {virt }}=N_{\text {track }} \frac{\eta}{\xi} \frac{3 A}{2 \pi R_{p}^{2}}
$$

where $A$ is the area of the two-dimensional domain that is initially seeded with particles.

Given the big difference in terms of density (we consider $\xi=\rho_{s} / \rho_{g}=\mathrm{O}\left(10^{3}\right)$ ) and the essentially inviscid nature of the large-scale flow (which does not display boundary layers adjacent to the solid walls), the tracked particles are assumed to undergo perfect elastic interactions when they collide with the walls.

\section{Space discretization}

We use the classical two-dimensional van Leer splitting over a uniform mesh. ${ }^{29}$ The basic idea behind this class of techniques is that the flux can be split into two components so that each may be properly discretized using upwind stencils to maintain stability and accuracy.

The following variant of flux splitting, in particular, is based on the Mach number splitting introduced by Laney. ${ }^{30}$ In that approach, the Mach number for supersonic flow is the full scalar Mach number in the downwind direction but zero in the upwind direction, whereas for subsonic flow it is given by $M^{ \pm}= \pm 1 / 4(M \pm 1)^{2}$ for $-1<M<1$. Such an approach results in

$$
\underline{F}=\rho_{g} a\left[\begin{array}{c}
\frac{u}{a} \\
a\left(\frac{u^{2}}{a^{2}}+\frac{1}{\gamma}\right) \\
a\left(\frac{u v}{a^{2}}\right) \\
a^{2} \frac{u}{a} \frac{(\gamma-1)\left(u^{2}+v^{2}\right)+2 a^{2}}{2(\gamma-1) a^{2}}
\end{array}\right] \quad \underline{G}=\rho_{g} a\left[\begin{array}{c}
\frac{u}{a} \\
a\left(\frac{u v}{a^{2}}\right) \\
a\left(\frac{v^{2}}{a^{2}}+\frac{1}{\gamma}\right) \\
a^{2} \frac{v}{a} \frac{(\gamma-1)\left(u^{2}+v^{2}\right)+2 a^{2}}{2(\gamma-1) a^{2}}
\end{array}\right]
$$

for supersonic flow and 


$$
\underline{F}^{ \pm}= \pm \frac{\rho_{g}}{4 a}(u \pm a)^{2}\left[\begin{array}{c}
\frac{(\gamma-1) u \pm 2 a}{\gamma} \\
v \\
\frac{v^{2}}{2}+\frac{[(\gamma-1) u \pm 2 a]^{2}}{2\left(\gamma^{2}-1\right)}
\end{array}\right] \quad \underline{G}^{ \pm}= \pm \frac{\rho_{g}}{4 a}(v \pm a)^{2}\left[\begin{array}{c}
1 \\
u \\
\frac{(\gamma-1) v \pm 2 a}{\gamma} \\
\frac{u^{2}}{2}+\frac{[(\gamma-1) v \pm 2 a]^{2}}{2\left(\gamma^{2}-1\right)}
\end{array}\right]
$$

for subsonic flow ( $a$ being the local speed of sound and $\gamma$ the ratio of the specific heat coefficients). According to this decomposition, as shown by eq. (12), the full flux for left-to-right supersonic flow $(M>1)$ is a function of variables solely from the left (plus), whereas the full flux for right-to-left supersonic flow $(M<-1)$ is a function of variables solely from the right (minus). For a subsonic flow (eq. 13), the flux will be a combination of both plus and minus flux contributions, with each component appropriately stenciled. Such an arrangement is summarized in Eq. (14):

$\begin{array}{ll}\mathrm{M} \geq 1 & \underline{F}=\underline{F}\left(\underline{q}_{\text {left }}\right), \\ \mathrm{M} \leq-1 & \underline{F}=\underline{F}\left(\underline{q}_{\text {right }}\right), \\ -1<\mathrm{M}<1 \quad \underline{F}=\underline{F}^{+}\left(\underline{q}_{\text {left }}\right)+\underline{F}^{-}\left(\underline{q}_{\text {rightt }}\right) .\end{array}$

\section{$\underline{\text { E. Time Integration }}$}

We apply an explicit MacCormack scheme directly to Eq. (3). Such a method is articulated essentially into two macroscopic steps:

Predictor step

$\underline{q}_{i, j}^{*}=\underline{q}_{i, j}^{n}-\frac{\Delta t}{\Delta x}\left(\underline{F}_{i+1, j}^{n}-\underline{F}_{i, j}^{n}\right)-\frac{\Delta t}{\Delta y}\left(\underline{G}_{i, j+1}^{n}-\underline{G}_{i, j}^{n}\right)-\Delta t \underline{S}_{i, j}^{n}$

Corrector step

$\underline{q}_{i, j}^{n+1}=\frac{1}{2}\left[\underline{q}_{i, j}^{n}+\underline{q}_{i, j}^{*}-\frac{\Delta t}{\Delta x}\left(\underline{F}_{i, j}^{*}-\underline{F}_{i-1, j}^{*}\right)-\frac{\Delta t}{\Delta y}\left(\underline{G}_{i, j}^{*}-\underline{G}_{i, j-1}^{*}\right)\right]-\Delta t \underline{S}_{i, j}^{n}$

The resulting scheme has second-order accuracy in both time and space. Following Anderson ${ }^{31}$, this scheme is "corrected" via the addition of fourth-order numerical dissipation terms computed as

$$
\varepsilon(|u|+a) \frac{p_{i+1, j}+p_{i-1, j}-2 p_{i, j}}{p_{i+1, j}+p_{i-1, j}+2 p_{i, j}}\left(\underline{q}_{i+1, j}-2 \underline{q}_{i, j}+\underline{q}_{i-1, j}\right),
$$


$\varepsilon(|v|+a) \frac{p_{i, j+1}+p_{i, j-1}-2 p_{i, j}}{p_{i, j+1}+p_{i, j-1}+2 p_{i, j}}\left(\underline{q}_{i, j+1}-2 \underline{q}_{i, j}+\underline{q}_{i, j-1}\right)$.

The fourth-order nature of these terms, which are added to improve the overall algorithm performances in relation to stability and presence of spurious oscillations, can be seen in the numerators, which are products of two second-order central-difference expressions for second derivatives ( $\varepsilon$ being an arbitrarily small value, typically of $\mathrm{O}\left(10^{-2}\right)$ ).

Similar care is devoted to the particle tracking equation, which is integrated using a standard fourthorder accurate explicit Runge-Kutta method.

Because the overall approach is based on an explicit formulation, the time step must be properly constrained by a stability criterion. In particular, we use the following version of the CourantFriedrichs-Lewy (CFL) criterion, where $a_{i, j}$ is the local value of the speed of sound and $K$ is the Courant number $(0.5 \leq K \leq 0.8)$ :

$$
\begin{aligned}
& \left(\Delta t_{C F L}\right)_{i, j}=\left[\frac{\left|u_{i, j}\right|}{\Delta x}+\frac{\left|v_{i, j}\right|}{\Delta y}+a_{i, j} \sqrt{\frac{1}{\Delta x^{2}}+\frac{1}{\Delta y^{2}}}+2 \zeta_{i, j}\left(\frac{1}{\Delta x^{2}}+\frac{1}{\Delta y^{2}}\right)\right]^{-1}, \\
& \zeta_{i, j}=\max \left\{\frac{4 \gamma \mu_{i, j}^{2}}{3 \operatorname{Pr} \rho_{i, j}}\right\}, \\
& \Delta t=\min \left\{K\left(\Delta t_{C F L}\right)_{i, j}\right\} .
\end{aligned}
$$

\section{F. Algorithm validation: the shock-tube problem}

To be in a position to obtain reliable information about the dynamics of interest, it is necessary to rely on a model that has been duly validated against other existing data. For a check on the validity of our entire theoretical structure, in particular, we consider the canonical shock-tube problem in which the driven section of the shock tube (the region with lower pressure) is initially seeded with a monodisperse collection of stationary spherical particles, as shown in Fig. 1a. 


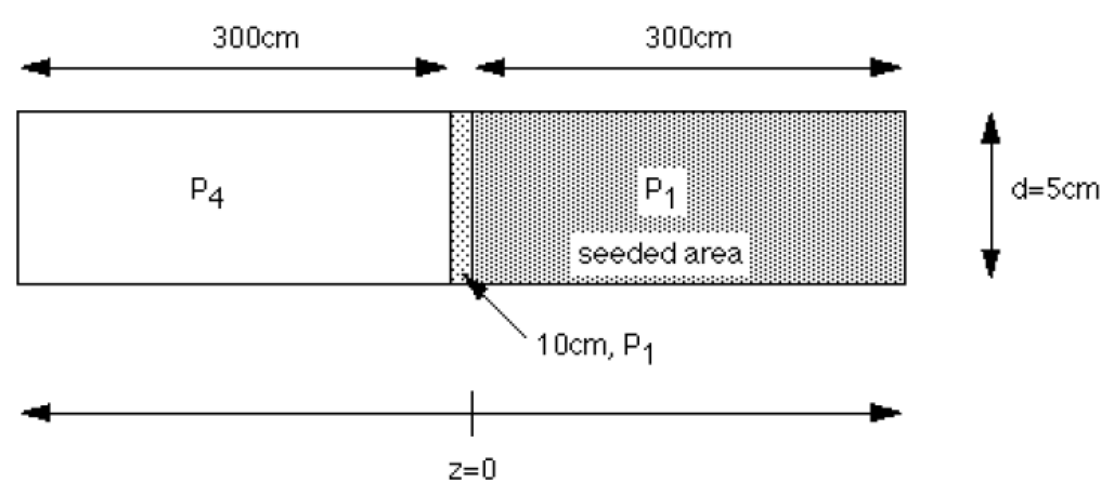

a)

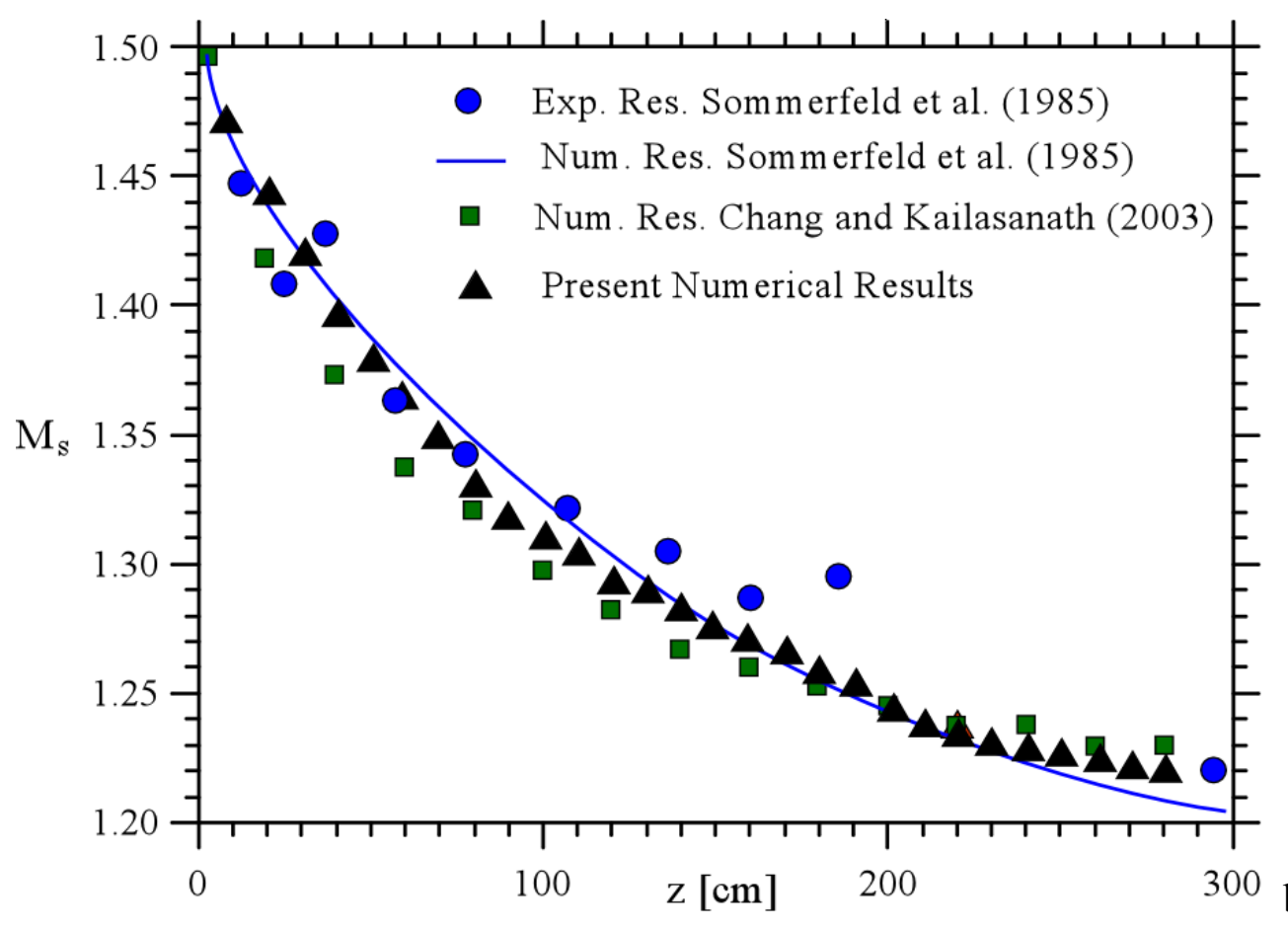

b)

Figure 1: Algorithm validation. Shock Mach number as a function of shock position inside the driven section of the shock tube: comparison with existing experimental and numerical results.

The dispersed region consists of $27-\mu \mathrm{m}$ diameter glass beads of density $\rho_{s}=2.5 \mathrm{~g} / \mathrm{cm}^{3}$ suspended in air at pressure $p=1 \mathrm{~atm}$. The mass loading, given as the ratio of the mass of the dispersed phase to the mass of the gas phase in the driven section, is fixed to $\eta=0.63$. The initial velocity of the shock wave is such that its Mach number is $M_{s}=1.49$. As can be seen in Fig. 1b, the results from the current simulation with 6,000 points in the $x$ direction and 50 points in the $y$ direction are in very good agreement with those (both experimental and numerical) presented by Sommerfeld ${ }^{6}$ though they slightly overestimate those provided later by Chang and Kailasanath ${ }^{5}$ (among other things, the good agreement between the present numerical results and the experimental ones by Sommerfeld ${ }^{6}$ justifies our decision not to consider in our model the heat exchange between fluid and particles, expected to be negligible in comparison to the exchange of kinetic energy). 


\section{G. Initial conditions for the shock-in-a-box case}

In this section, we finally turn to the specific problem under investigation here. In particular, insights are sought by considering a relatively simple archetypal setting corresponding to a shock wave originating from a small region located inside a closed cavity containing particles dispersed in the fluid. Such a configuration is considered as a relevant model of the explosion dynamics produced by the sudden release of a significant amount of chemical, nuclear, electrical, or mechanical energy in a limited space. The choice of the initial conditions that make such a problem well posed from both a physical and computational point of view is not as straightforward as one would imagine and requires some physical reasoning.

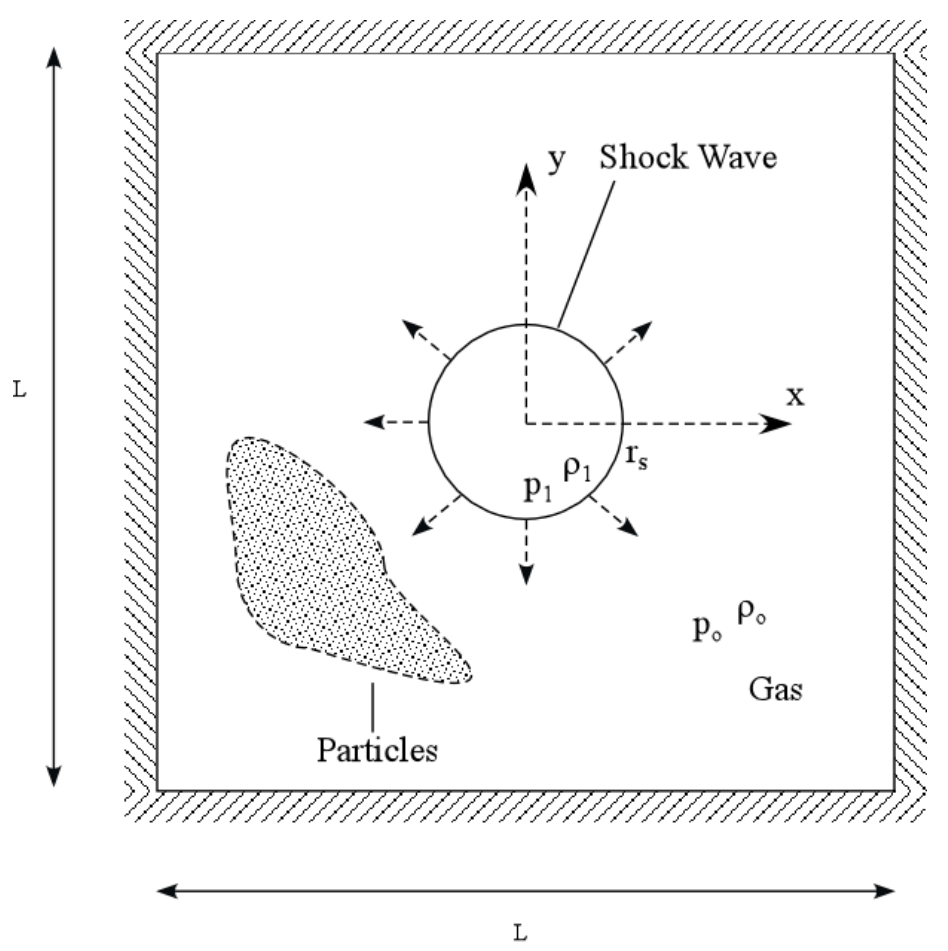

Figure 2: Sketch of the considered problem. A strong circular shock moves into the mixture of gas and particles at rest, imparting a radial, outward velocity on the gas and particles as it passes over.

We assume a very hot region to be located at the center of the domain, encompassing very low density and finite pressure. We imagine such a hot region of finite size (radius $r_{s}$ ), present in the computational domain at the initial simulation time $t=0$, to be the result of a shock ideally starting with an infinite strength at the exact origin $(x=0, y=0$, see Fig. 2$)$ of the reference system and then expanding radially (while undergoing a simultaneous decrease in strength).

With the pre-shock state corresponding to the properties of the undisturbed gas $\left(p_{o}, \rho_{o}\right)$, we determine the post-shock thermodynamic state using the standard Rankine-Hugoniot conditions for a travelling shock:

$$
\rho_{1}=\rho_{o} \frac{(\gamma+1) M_{s}^{2}}{2+(\gamma-1) M_{s}^{2}},
$$




$$
\begin{aligned}
& p_{1}=p_{o}\left[1+2 \frac{\gamma}{(\gamma+1)}\left(M_{s}^{2}-1\right)\right], \\
& V_{1}=M_{s} \sqrt{\gamma \frac{p_{o}}{\rho_{o}}\left(1-\frac{\rho_{o}}{\rho_{1}}\right)},
\end{aligned}
$$

where $M_{S}$ is the shock-wave Mach number and $V_{1}$ is the radial velocity imparted by the shock wave to the fluid at $r=r_{s}$.

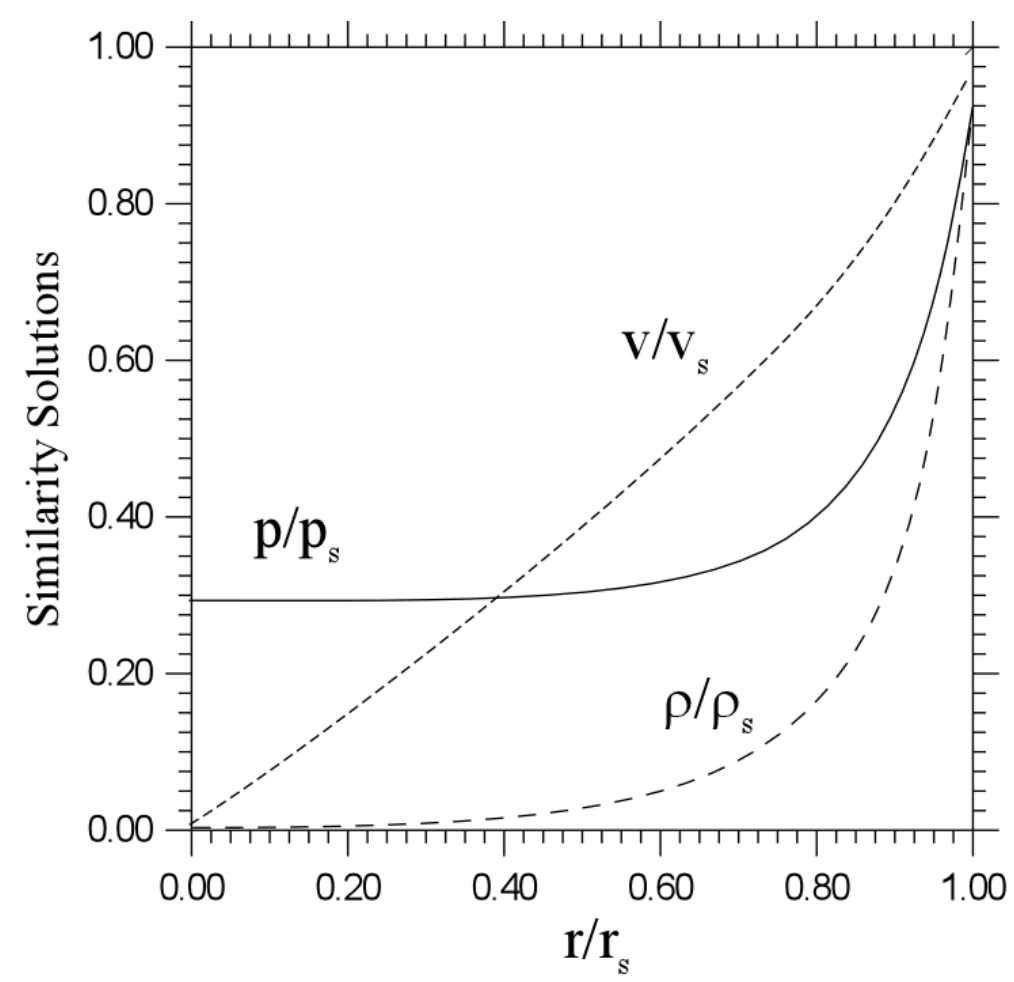

Figure 3: Nondimensional density, pressure and radial velocity profiles inside the ball of initial conditions delimited by the traveling shock wave. After an initial transient period, all independent variables (normalized to their shock values) approach a self-similar variation between the origin and the shock.

To initialize the thermofluid dynamics inside the "ball" of initial conditions delimited by the circular shock wave located at $r=r_{s}$, we use the "similar solutions" developed by Sakurai. ${ }^{32}$ On the basis of such solutions, which represent the behavior of shock waves originating ideally from an infinitesimal (point-source) region, all the thermofluid-dynamic variables can be assumed to approach, after a short initial startup period, a "universal profile" between the origin and the shock. Sakurai $^{32}$ determined such solutions using a well-known technique already used by other authors in companion fields of fluid dynamics (such as boundary-layer theory and conical-flow theory). In practice, the assumption of similarity decreases the number of independent variables, thereby reducing the partial differential equations underlying the full nonlinear hydrodynamic problem to a set of ordinary differential equations. 
However, the applicability of such solutions requires that the thermofluid-dynamic variables are properly nondimensionalized. As shown in Fig. 3, they must be put in non-dimensional form using the corresponding shock values for the thermodynamic properties and the radial position of the shock as a reference length. In this way, the problem is further simplified by turning the moving boundary condition at the shock front into a much more manageable limit-value condition. This leads to the "self-similar" profiles shown in Fig. 3.

As the reader will easily realize by taking a look at them, the radial velocity can be assumed to undergo an almost linear decrease from its shock value to zero at the origin (the gas velocity at the origin of the blast is zero and remains so while it is undisturbed). A region of very hot, low-density gas is left near the origin of the explosion.

We initially place the shock at a radial distance $r_{s}=0.1 L$ from the origin of the blast. Particles are seeded uniformly only outside this region, assuming that the shock wave developed through a particle-free region during its startup period.

As a concluding remark for this section, we justify our decision to consider the "full domain" shown in Fig. 2 (entire square box) - rather than the quarter portion that could have been selected on the basis of relatively simple considerations of the vertical and horizontal symmetries of the considered problem - by the advantage that it affords in assessing the eventual role of particles in the onset of possible fluid-dynamical instabilities. Such instabilities arise during the interaction of shock waves with inhomogeneities ${ }^{1,33}$ and may be responsible for significant symmetry-breaking phenomena.

\section{Results}

\section{A. Problem description}

As illustrated schematically in Fig. 2, the enclosure is a square of size $L=1 \mathrm{~m}$ and the initial, perfectly symmetric blast is modeled by resorting to the aforementioned self-similar solutions developed originally by Sakurai. ${ }^{32}$ As the unperturbed (pre-shock) conditions, we consider standard air $(\gamma=1.4$, Prandtl number $P r=0.71)$ in quiescent conditions with pressure $p_{o}=1$ atm and density $\rho_{o}=1.225 \mathrm{~kg} / \mathrm{m}^{3}$. The simplicity of the geometry and its boundary conditions make the former an ideal case in which to study parametrically the effects induced by dispersed solid mass. Although two-dimensional simulations may not capture the underlying physics quantitatively, our findings will help to clarify controversial experimental observations and elucidate the underlying physical mechanisms.

To track the system evolution, several solid particles are initially seeded uniformly into the area delimited by the shock wave and the external solid boundaries. In particular, we make sure such a number is statistically relevant (see Sect. IIIB for additional information on this specific point). The related number of virtual particles employed is chosen appropriately in order to achieve the desired mass loading, as explained in Sect. IIC. 
The mass density of the particles is assumed to be fixed $\left(\rho_{\mathrm{s}}=10^{3} \mathrm{Kgm}^{-3} \rightarrow \xi=816\right)$. However, several degrees of freedom are introduced in the problem by varying parameters that are related to the solid particles or the shock wave. The mass loading is allowed to span the relatively large range of $0 \leq \eta \leq 4$, with $\eta=0$ representing the trivial case in which the propagation of the shock wave is unaffected by the particles (such a condition being considered essentially for the sake of comparison with the other cases for which $\eta \neq 0$ ). We also change parametrically the "energy" of the blast by tuning the initial strength of the shock wave via the related value of $M_{s}$ (provided as an input parameter to the algorithm).

Furthermore, for each of the above cases, the characteristic particle size is varied in the range 12.5 $\leq R_{p} \leq 50 \mu \mathrm{m}$ to account for another effect: the expected influence exerted on the dynamics by the overall "particle surface area" exposed to the gas.

Along these lines, we recall that particles exert their influence on the propagation of the shock wave essentially through two different effects: one of an "inertial" nature that depends on the total amount of solid mass physically present in the fluid domain, and the other of an essentially "viscous" nature due to the "friction" between the particles and the gas itself.

Proper parametric variation of all such quantities allows us to explore the full set of dynamics resulting from the interaction of waves having different initial energies with fluid-particle mixtures that possess different properties in relation to inertial and drag responses.

\section{$\underline{\text { B. Grid-refinement study }}$}

With the numerical approach used here, a $300 \times 300$ Cartesian grid was found to be sufficient for attaining conditions of mesh-independency. This statement is based on an a priori grid tailoring study that was carried out expressly to quantify the improvement of resolution achievable by mesh adaptation. In that preparatory study, we scaled the number of particles effectively tracked for each simulation with the mesh $\left(N_{\text {track }}=N_{x} \times N_{y}\right)$ while maintaining constant the number of virtual particles required to have a fixed value of the mass load (in particular, we assumed $\eta=2$ and $R_{p}=12.5 \mu \mathrm{m}$ for the grid-refinement study).

The results, summarized in Fig. 4 for both $M_{s}=4.5$ and 10, demonstrate the reliability of the present hybrid Eulerian-Lagrangian numerical approach and the related "virtual-particle" philosophy. By scaling the number of points used for the Eulerian uniform structured mesh and the number of tracked Lagrangian particles accordingly, a threshold could be identified above which the solution became independent of both the mesh and the number of tracked particles.

Although a $300 \times 300$ mesh would have been sufficient to ensure grid independency and high accuracy (the curves related to grids with $300 \times 300,400 \times 400$, and $500 \times 500$ points are nearly indistinguishable), we decided to conduct all the simulations using a $500 \times 500$ mesh given the better quality achievable by using such a grid for the graphical representation of the propagation velocity of the shock (although not explicitly required by the algorithm, the shock speed has been used to elaborate the arguments discussed in Sect. IIIA). 

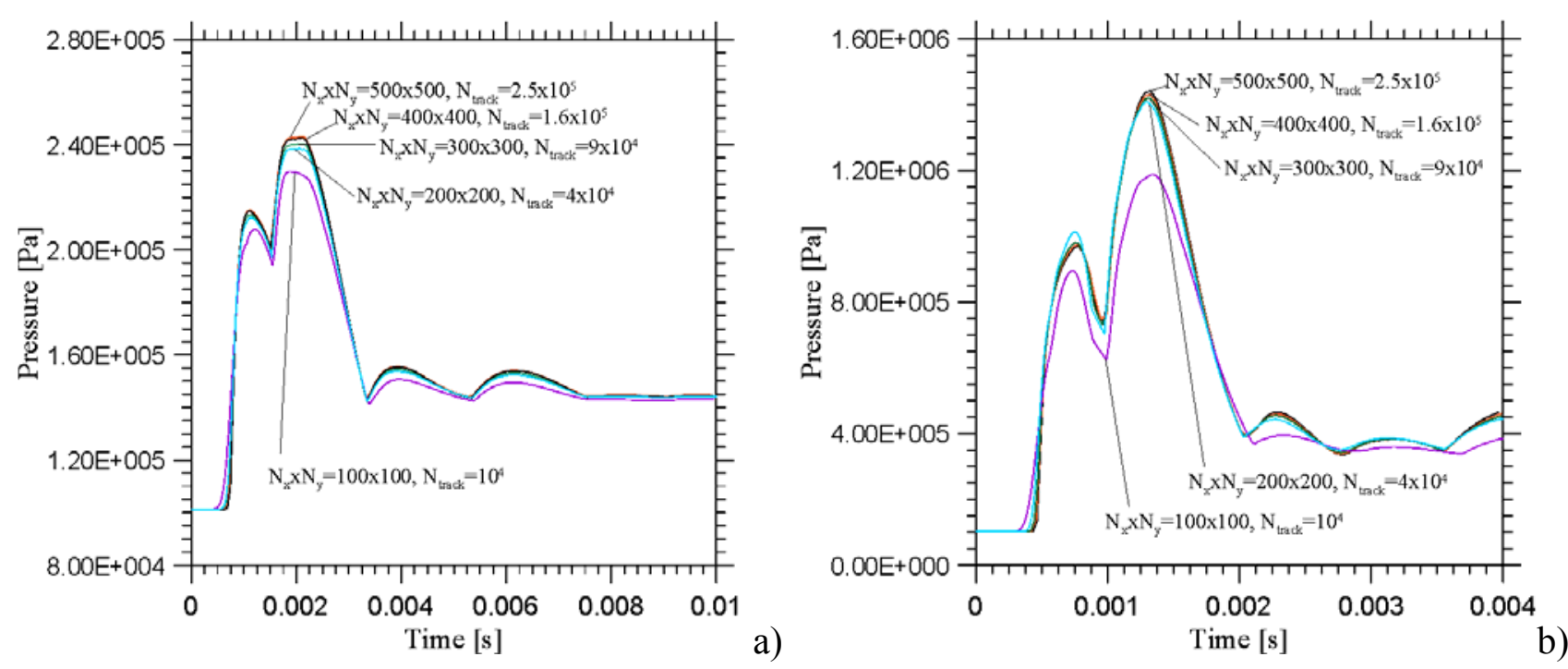

Figure 4: Grid-refinement study and verification of the virtual-particle approach. Maximum pressure at the solid wall as a function of time for $\eta=2$ and $R_{p}=12.5 \mu \mathrm{m}$ : (a) $M_{s}=4.5$; (b) $M_{s}=10$.

With such choices of physical and geometrical (size) system properties and numerical discretization, the two criteria defined in Sect. IIB and IIC for the conditions to be satisfied to make our overall mathematical and numerical approach applicable (well-posed) are largely met with both St and $\phi=\frac{2}{3} \pi R_{p}^{2} / \Delta x \Delta x$ being much smaller than unity. For the considered conditions and mesh, $3.5 \times 10^{-}$ ${ }^{11} \leq S t \leq 5.5 \times 10^{-10}$ and $8 \times 10^{-5} \leq \phi \leq 1.3 \times 10^{-3}$; such conditions are required to ensure the validity of the particle tracking equation and the related two-way coupling modeling.

In the discussion below, the results are presented in terms of the propagation velocity of the shock wave as a function of its distance from the center of the cavity (Figs. 5 and 6), the pressure loads on the walls of the box as a function of time (as shown in Fig. 4), and complete spatial patterns that show the effective distribution of the thermofluid-dynamic variables and particles inside the enclosure at different times. The evolution of the system is presented essentially in terms of pressure contour maps (Figs. 11-14).

\section{Shock-wave propagation velocity}

To provide some immediate insights into the effect of particles on the dynamics under consideration, we begin by concentrating on the very early stage of evolution. This is in relation to the time required by the shock wave originating from the central circular region to first "touch" the external boundary, i.e. to cover a distance of exactly $L / 2$ from the center of the domain. We will address the ensuing complex reflection phenomena due to the interaction with the walls in the next two sections. 
The key aim here is to introduce some predictive links between the properties of the resulting shock wave and those of the particles. Related results are summarized in Figs. 5 and 6, which show for $M_{s}=10$ (the trends for $M_{s}=4.5$ being qualitatively similar) the changes experienced by the shock velocity for increasing values of the mass load $\eta$ (fixed particle radius) and particle radius $R_{p}$ (fixed mass load), respectively.

The fundamental mechanism by which energy is channeled from the fluid into the particles (or vice versa) is reasonably well understood and allows the problem to be cast in terms of a well-defined set of equations as illustrated in the preceding sections. However, the effective nonlinear dynamics displayed by such processes are still largely unknown. Figure 5 reveals the increasing attenuation of the amplitude of the gas-phase velocity that can be obtained for a fixed value of the particle radius when the mass dispersed in the gas is increased. Indeed, the Mach number attained by the shock wave shortly before it meets the solid boundary undergoes a percentage decrease with respect to its unperturbed (no particles) value ranging from $44 \%$ of for $\eta=2$ to approximately $53 \%$ for $\eta=4$.

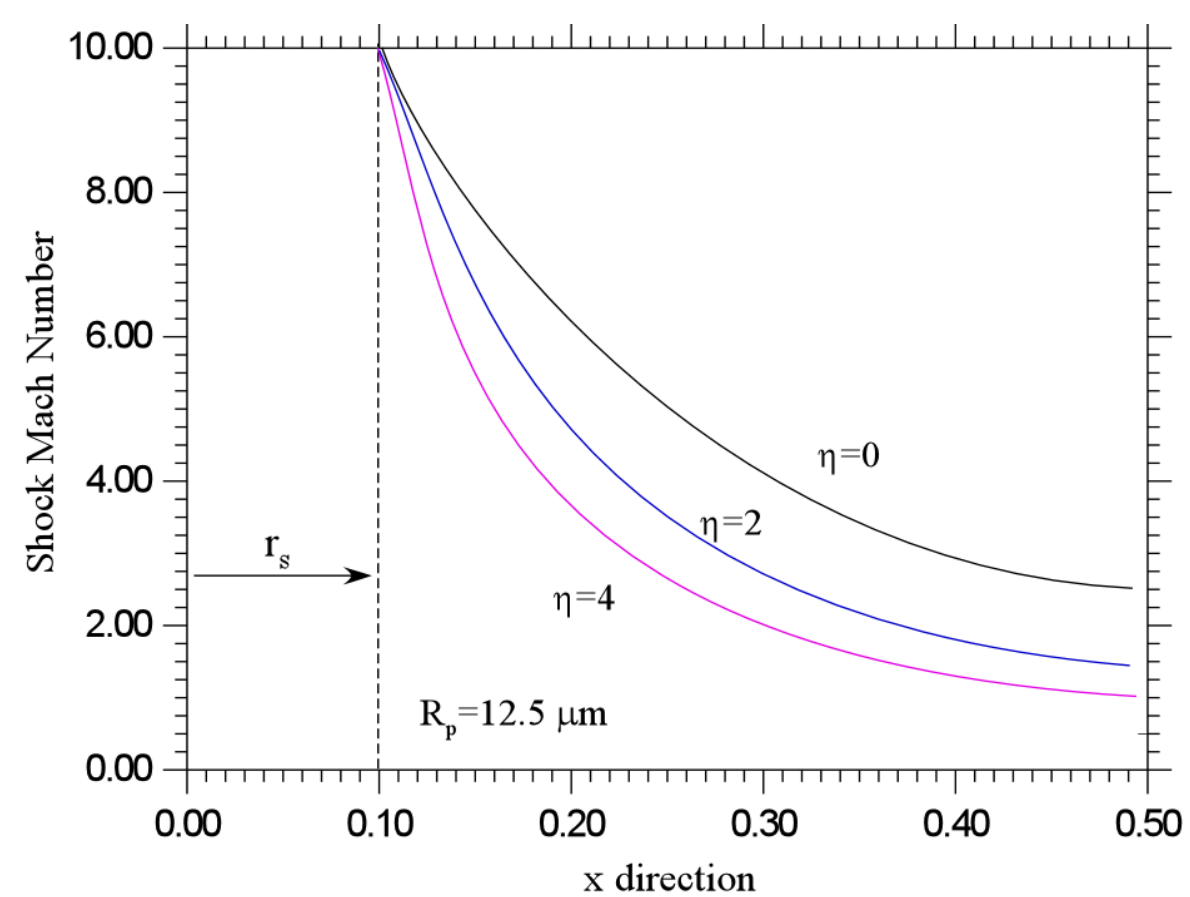

Figure 5: Shock-wave Mach number as a function of the distance from the center of the cavity for different values of the mass load at a fixed value of the particle radius $\left(M_{s}=10, R_{p}=12.5 \mu \mathrm{m}\right)$. The solid, continuous lines were obtained via a fourth-order polynomial expression interpolating the underlying numerical data. 


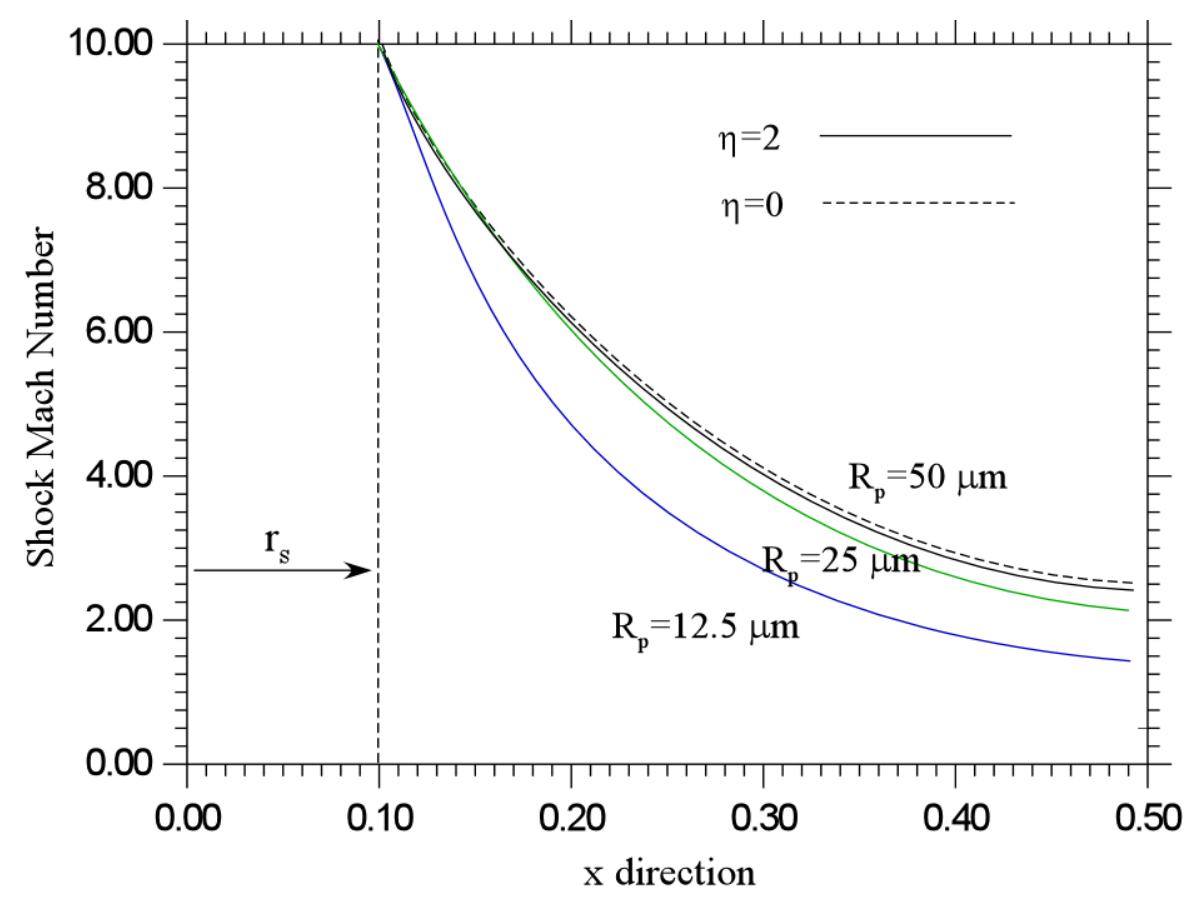

Figure 6: Shock-wave Mach number as a function of the distance from the center of the cavity for different values of the particle radius at a fixed value of the mass load $\left(M_{s}=10, \eta=2\right)$. The solid, continuous lines were obtained via a fourth-order polynomial expression interpolating the underlying numerical data.

The results in Fig. 6 are even more interesting, in that they provide evidence for the role of the viscous interactions between the gas and the particles. While inertial effects are expected to depend solely on the total amount of solid mass physically added to the fluid system, the influence of such viscous interactions is much more subtle because it depends on the overall number of particles and their effective radius.

As shown in Fig. 6, for a fixed value of $\eta$, the larger the particles, the less significant the decrease displayed by the shock-wave Mach number. Indeed, the $44 \%$ decrease experienced by the Mach number of the shock wave just before it hits the solid boundary for $R_{p}=12.5 \mu \mathrm{m}$ is reduced to only $15 \%$ for $R_{p}=25 \mu \mathrm{m}$. In practice, a particle-size threshold can be identified above which the influence of dispersed mass becomes more or less negligible over the considered time frame (although the reader may notice a small departure as time progresses, the curve for $R_{p}=50 \mu \mathrm{m}$ overlaps almost perfectly with that obtained for $\eta=0$ ).

Such observations will prove very useful for interpreting the results in the subsequent sections, the main focus of which is the diversity of fascinating patterns that are produced by the complex interplay among the multiple shock reflections and particle-fluid and particle-wall interactions. 


\section{Pressure load}

This section is devoted to examining another important aspect embedded in the considered problem, namely the specific behavior displayed by the pressure at the walls (such information being very important in the context of the aforementioned engineering applications related to the assessment of structural resistance to explosive loads).

We examine these aspects by plotting the maximum pressure at the walls as a function of time (Figs. 7 and 9) and its spatial distribution along the wall at selected instants (Figs. 8 and 10). We show how the problem becomes more intuitive and manageable when cast in the form of pressure-wall behavior and related physical connections with particle-dependent mechanisms and effects.

Apart from providing meaningful and useful information from an engineering standpoint, such plots can also be used to better characterize the multiple shock reflections that occur inside the cavity as time passes. Indeed, each of the "peaks" visible in Figs. 7 and 9 corresponds to a shock reflection.

Taken together, such plots clearly indicate that the most evident and immediately realizable effect of particles is a remarkable decrease in the wall pressure load, the greatest variation in which is seen for the smallest particles considered $\left(R_{p}=12.5 \mu \mathrm{m}\right)$. Indeed, the peaks in Figs. 7 and 9 are significantly damped (the quantitative details related to the "first" wall shock reflection are reported in Table I).

Table I: Percentage variation experienced by the pressure load related to the first shock reflection in relation to initial shock-wave Mach number, particle radius, and mass load.

\begin{tabular}{|l|l|l|l|l|l|l|l|l|l|l|l|l|}
\hline$M_{s}$ & 4.5 & 4.5 & 4.5 & 4.5 & 4.5 & 4.5 & 10 & 10 & 10 & 10 & 10 & 10 \\
\hline$\eta$ & 2 & 2 & 2 & 4 & 4 & 4 & 2 & 2 & 2 & 4 & 4 & 4 \\
\hline $\begin{array}{l}R_{p} \\
(\mu \mathrm{m})\end{array}$ & 12.5 & 25 & 50 & 12.5 & 25 & 50 & 12.5 & 25 & 50 & 12.5 & 25 & 50 \\
\hline$\Delta \%$ & $65 \%$ & $28.5 \%$ & $\cong 0 \%$ & $66 \%$ & $50 \%$ & $\cong 0 \%$ & $66 \%$ & $41 \%$ & $\cong 0$ & $66 \%$ & $58 \%$ & $\cong 0$ \\
\hline
\end{tabular}

A notable change can also be seen in the relative amplitude of the peaks following the first peak. Hereinafter, we will refer to each visible peak in the history of the pressure load simply as a reflection of order $1,2,3$, etc., with " 1 " corresponding to the first noticeable peak in the figures, for which related results have been summarized in Table I.

With particles present, the relative amplitudes of peaks of order larger than one are much smaller than those visible in the pressure history for $\eta=0$ (no particles). In some cases (e.g., $R_{p}=12.5 \mu \mathrm{m}$ ), they are either strongly mitigated or completely suppressed, leaving only the order-one peak clearly visible in the temporal pressure distribution. On one hand, this observation confirms that the multireflection dynamics are strongly sensitive to the presence of dispersed solid mass. On the other hand, it also provides precise information about the related time scales. 


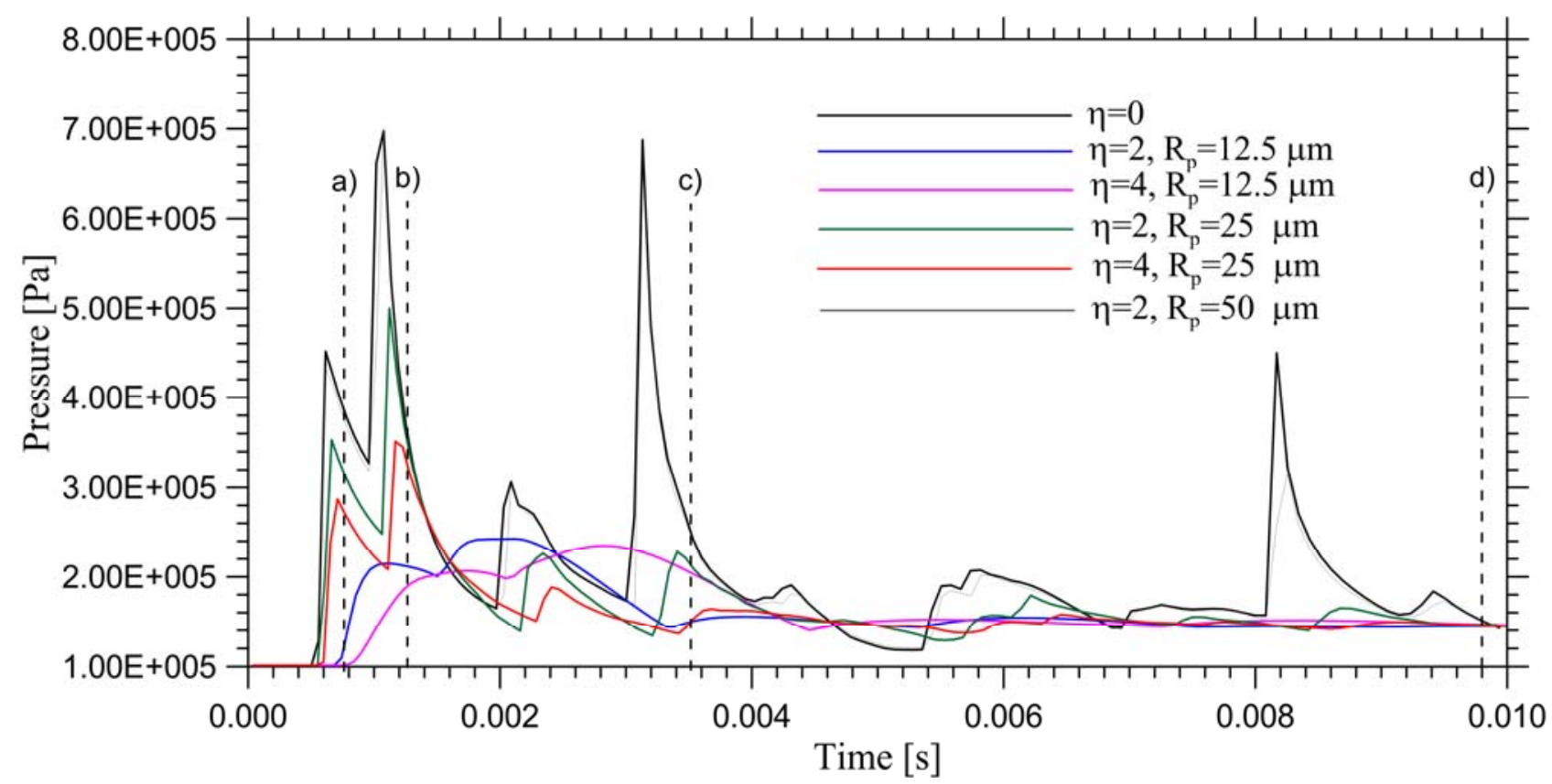

Figure 7: Maximum pressure at the solid wall as a function of time for $M_{s}=4.5$ and different values of mass load and particle size.
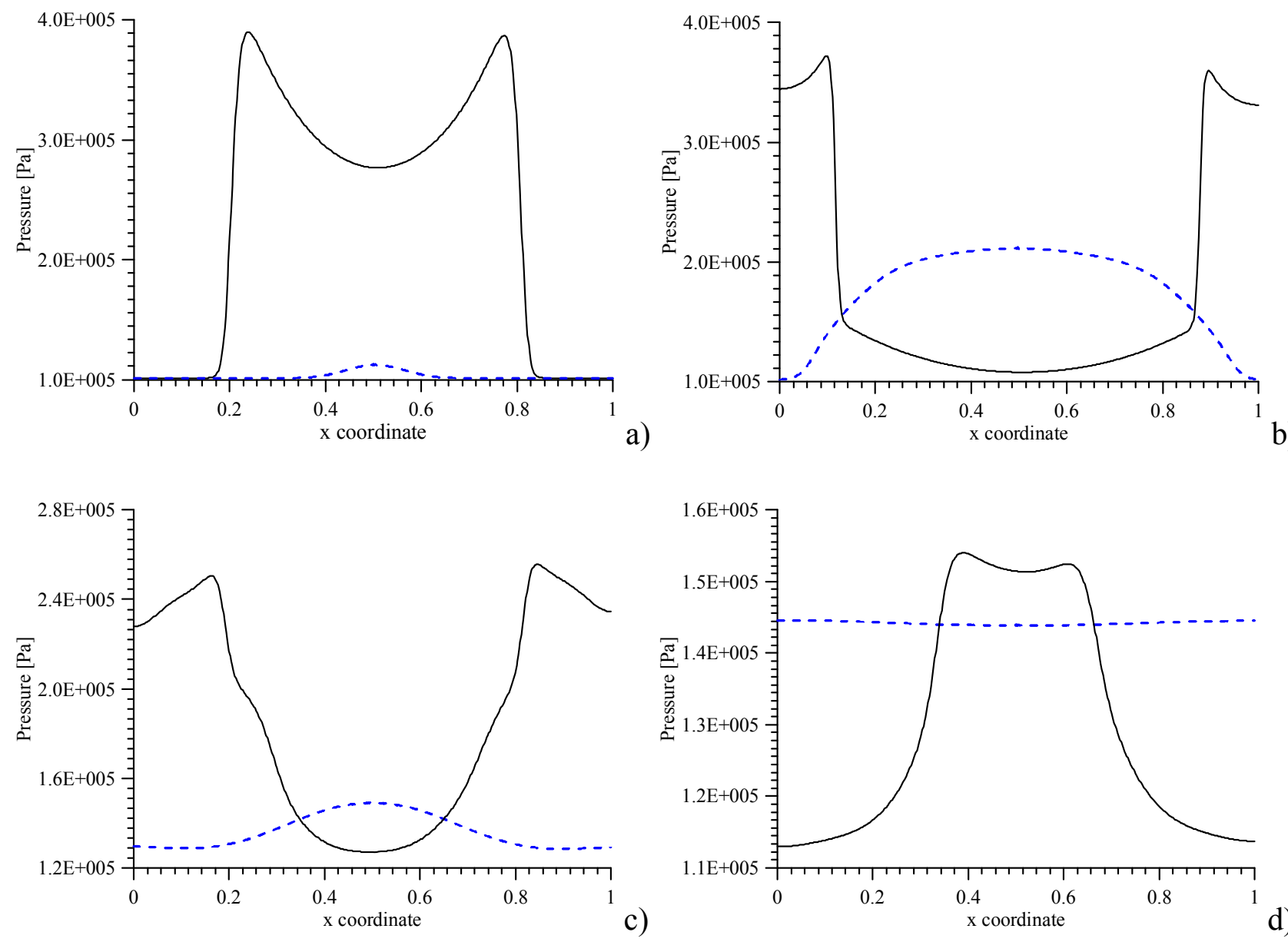

Figure 8: Distribution of pressure along the wall at different times (solid line: $M_{S}=4.5$, no particles; dashed line: $\eta=2, R_{p}=12.5 \mu \mathrm{m}$ ): (a) $t=7.5 \times 10^{-4} \mathrm{~s}$; (b) $t=1.25 \times 10^{-3} \mathrm{~s}$; (c) $t=3.5 \times 10^{-3} \mathrm{~s}$; (d) $t=1 \times 10^{-2} \mathrm{~s}$. 


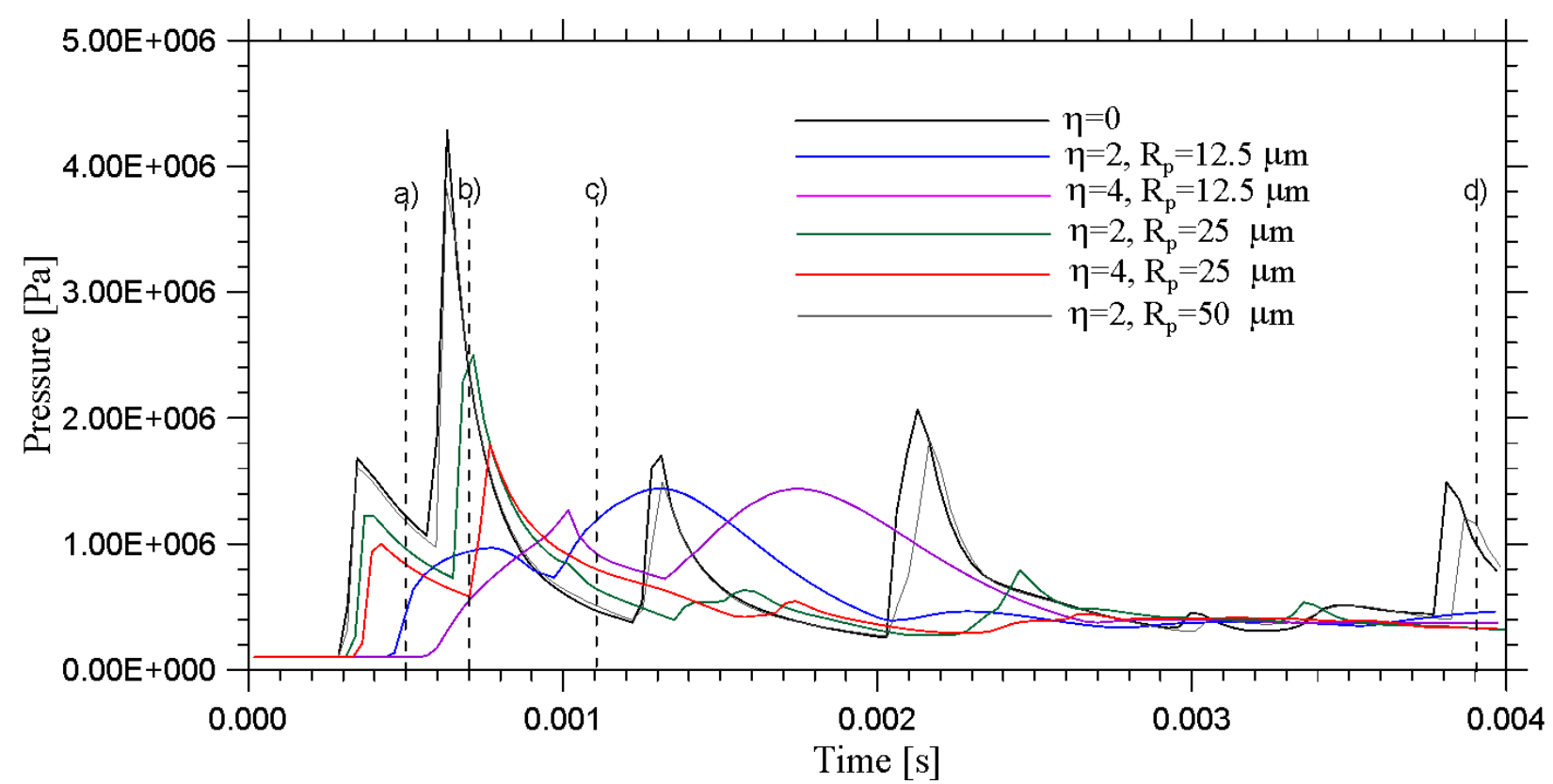

Figure 9: Maximum pressure at the solid wall as a function of time for $M_{s}=10$ and different values of mass load and particle size.
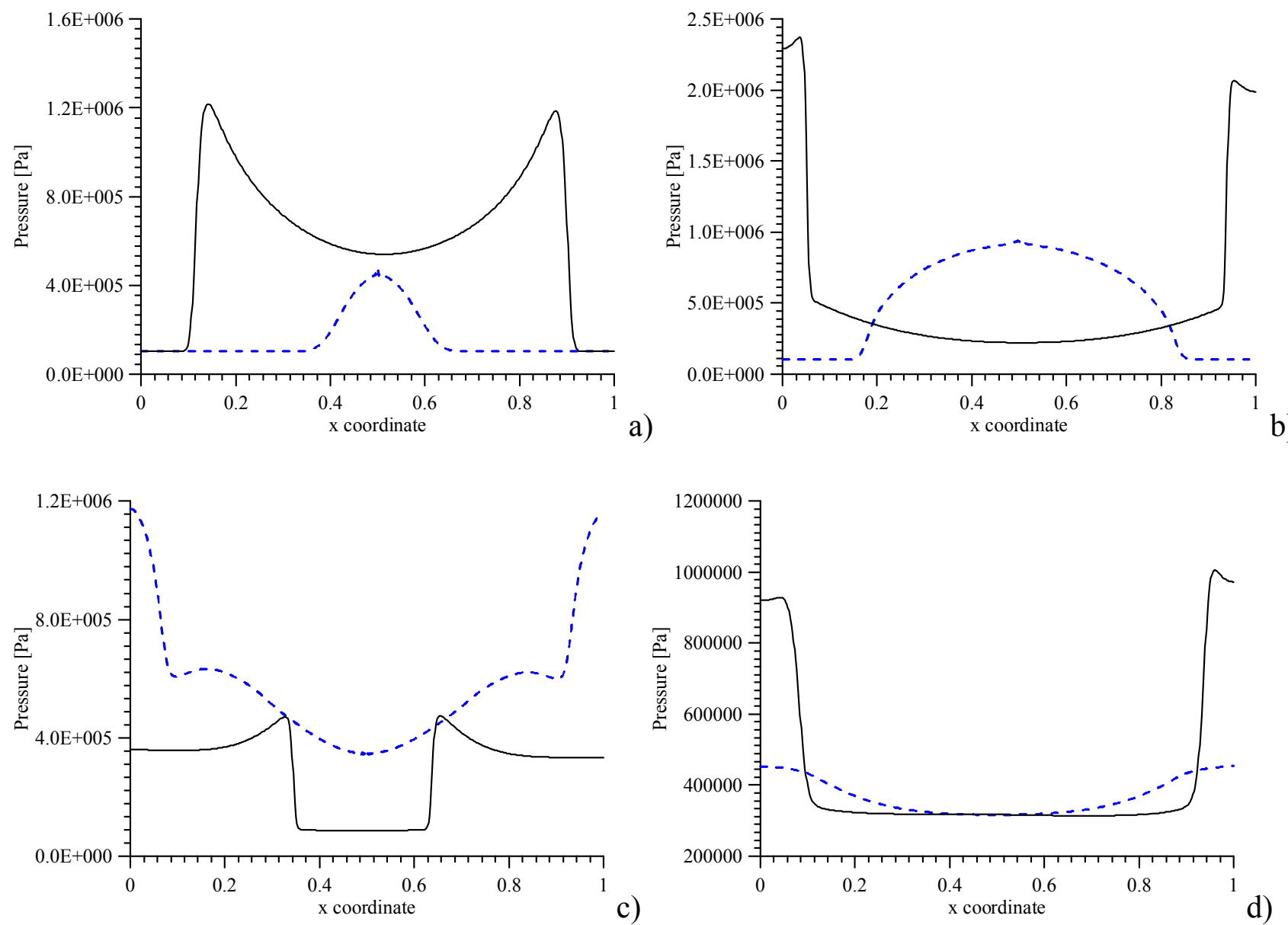

Figure 10: Distribution of pressure along the wall at different times (solid line: $M_{s}=10$, no particles; dashed line: $\eta=2, R_{p}=12.5 \mu \mathrm{m}$ ): (a) $t=5 \times 10^{-4} \mathrm{~s}$; (b) $t=7 \times 10^{-4} \mathrm{~s}$; (c) $t=1.1 \times 10^{-3} \mathrm{~s}$; (d) $t=4 \times 10^{-3} \mathrm{~s}$. 
As anticipated, such plots can also be used to obtain additional useful insights into the temporal evolution of the considered phenomena and related nonlinear dynamics. They show that when small particles are added to the gas, apart from the aforementioned decrease in intensity, the maxima of the curves tend to be shifted to the right of their initial positions (the peaks undergo a remarkable displacement toward larger times or later stages of evolution). Such an effect can be attributed to the decrease in velocity experienced by the shock waves, as seen in Figs. 5 and 6 and discussed in Sect. IIIC.

Along these lines, Figs. 7 and 9 are instrumental in unraveling the interwoven processes that would be overshadowed by limiting the analysis to the shock-wave propagation velocity alone. According to such plots, the peaks decrease more as the particles are made smaller, and the time shift increases with the mass load.

As a result of the two aforementioned effects (i.e., the mitigation of the peaks and their delayed appearance), the shock wave experiences significantly fewer reflections over a given observation timeframe than in the particle-free situation, the effective number of reflections depending on the specific situation considered.

Such arguments can also be used to explain the notable differences in the pressures at the end walls for $\eta=0$ and $\eta \neq 0$ shown in Figs. 8 and 10. The rationale can be found in the different time taken by the original (first) shock wave and related reflections of higher order to reach the cavity boundary. This time depends on the amount and radius of dispersed particles present in the fluid as summarized in Figs. 5 and 6.

Figures 8 and 10 show in detail that at specific times the pressure load on the wall is generally higher in the absence of particles. This is consistent with the realization that the shock wave traveling in the pure gas (be it the first shock wave or a reflection of higher order) has already reached the wall, whereas the corresponding shock wave traveling in the dusty gas has not reached the wall yet due to its reduced velocity. Another source of difference can be found obviously in the reduced intensity of the shock due to the presence of particles (as discussed above, the particles have a double effect on the shock wave by reducing both its velocity and intensity).

In line with the observations already reported in terms of shock-wave velocity (see Sect. IIIC), however, the role of dispersed solid mass tends to be increasingly less important as the particles are made larger. For $R_{p}=50 \mu \mathrm{m}$ (see Figs. 7 and 9), regardless of the considered mass load (over the range $0<\eta \leq 4)$, the curves for $\eta=0$ and $\eta \neq 0$ overlap both in terms of amplitude and stages of evolution (some perceptible decrease in the maximum wall pressure is still present because of the particles, but only in the much later stages of evolution).

By contrast, the effects produced by an increase in $\eta$ can be clearly seen for $R_{p}=25 \mu \mathrm{m}$ and $R_{p}=12.5 \mu \mathrm{m}$. For such values of the particle radius, larger values of $\eta$ produce an evident amplification of the time-shift trend affecting the peaks, as discussed before. However, the amplitude of peaks of order larger than one remains essentially the same when $R_{p}$ is kept constant. 
We can conclude from this that although a change in the particle size for a fixed value of the mass load $\eta$ does affect both the shock-wave strength and propagation velocity, modifying the mass load while keeping the particle size fixed tends to have more of an influence on the latter quantity.

Some final insights into the role played by the initial shock wave strength (namely, its initial Mach number) can be obtained by direct comparison of Figs. 7 and 9 and by considering again the quantitative data summarized in Table I. Interestingly, such analysis leads to the conclusion that an increase in the initial shock wave Mach number determines an amplification of the damping role exerted by particles on the pressure load for a given (fixed) value of the mass load. This can be clearly seen, e.g., for $R_{p}=25 \mu \mathrm{m}$. Indeed, the shrinkage experienced by the pressure load related to the first shock reflection for $\eta=2$ increases from $28.5 \%$ for $M_{s}=4.5$ to $41 \%$ for $M_{s}=10$. Similarly for $\eta=4$, the percentages are $50 \%$ and $58 \%$ for $M_{s}=4.5$ and $M_{s}=10$, respectively.

The present section leads on logically to the next one, in which the focus shifts from macroscopic quantities and "ensemble" trends of the system to the analysis of its microscopic details and patterning behavior as functions of space and time.

\section{E. Shock-wave reflection phenomena}

Following the approach of the preceding sections, we start from the simplest (and most instructive) case in which no particles are present in the gas (i.e. $\eta=0$ ). The related results, summarized in Figs. 11 and 12 for $M_{s}=4.5$ and 10, respectively, show immediately that some general qualitative features can be identified regardless of the specific value of $M_{s}$ considered. The related stages of evolution for $\eta=0$ (no particles) can be described as follows.

As soon as the outward traveling shock wave originating from the "ball" of initial conditions meets the solid boundary (Figs. 11a and 12a), four concave shock segments reflect from the four walls. Accordingly, four regions of high pressure are created in proximity to the boundary of the enclosure. As time passes, such regions of increased pressure expand laterally (the shock segments spread along the wall toward the corners). They also expand back towards the center of the explosion.

As a natural consequence, at a certain time that depends on the initial value of the shock Mach number, the reflected shock meets the exploded expansion wave at the center (the hot core), thereby giving rise to the onset of a new blast wave moving in the opposite direction (again toward the walls).

Continuing with the description of the qualitative features displayed by the pressure field, it is also worth highlighting that the related patterning behavior is made even more complex by the phenomena that occur in the corners of the cavity. Given that they are slightly farther from the center of the enclosure than are the central portions of the walls, such regions are reached slightly later by the outward-travelling shock waves. This effect results in shocks emerging from the corners later than the inward-travelling shocks reflected from intermediate points (among other things, this is also the root cause of the double-cusp feature of the "first" peak visible in Figs. 7 and 9). 

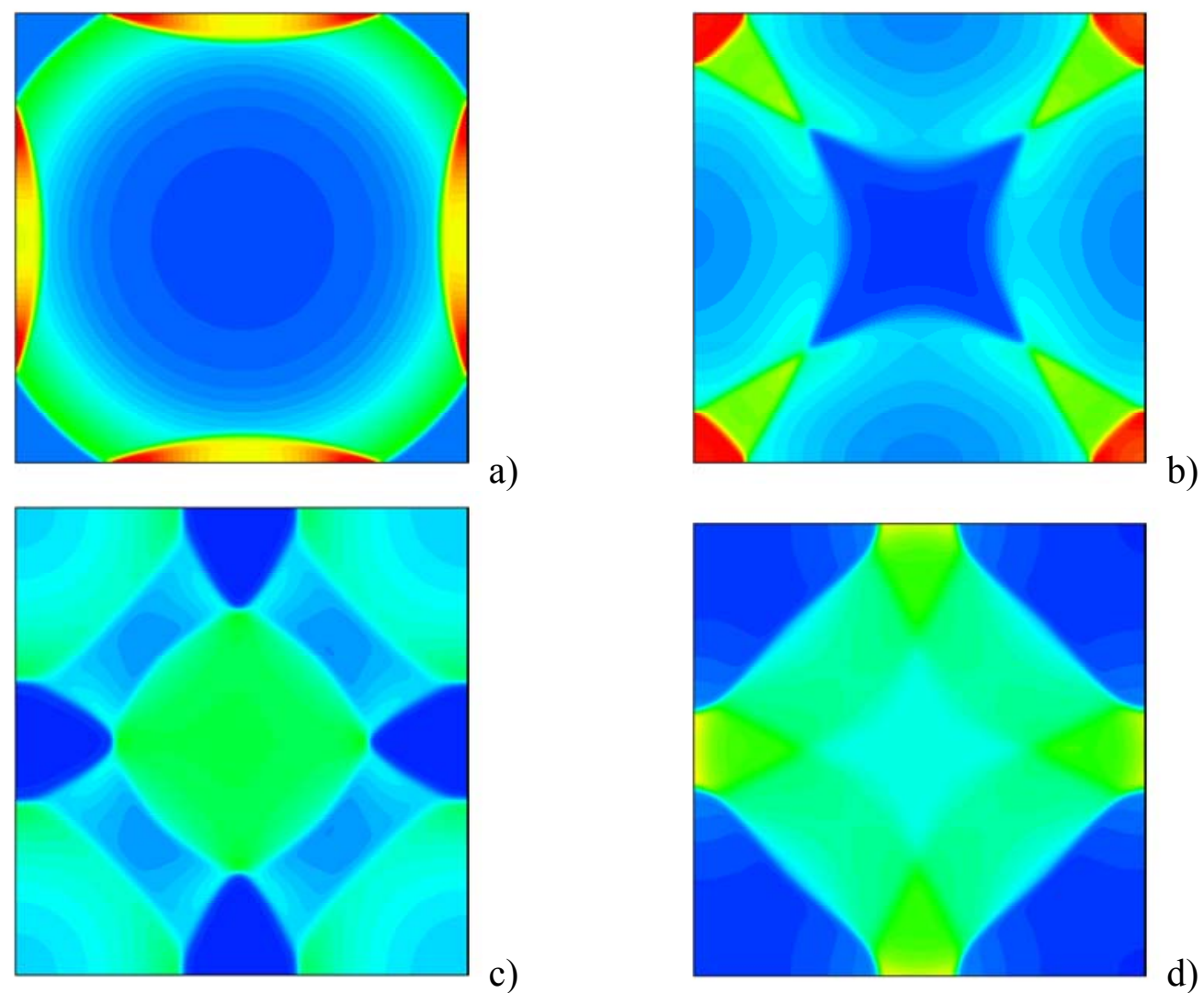

d)
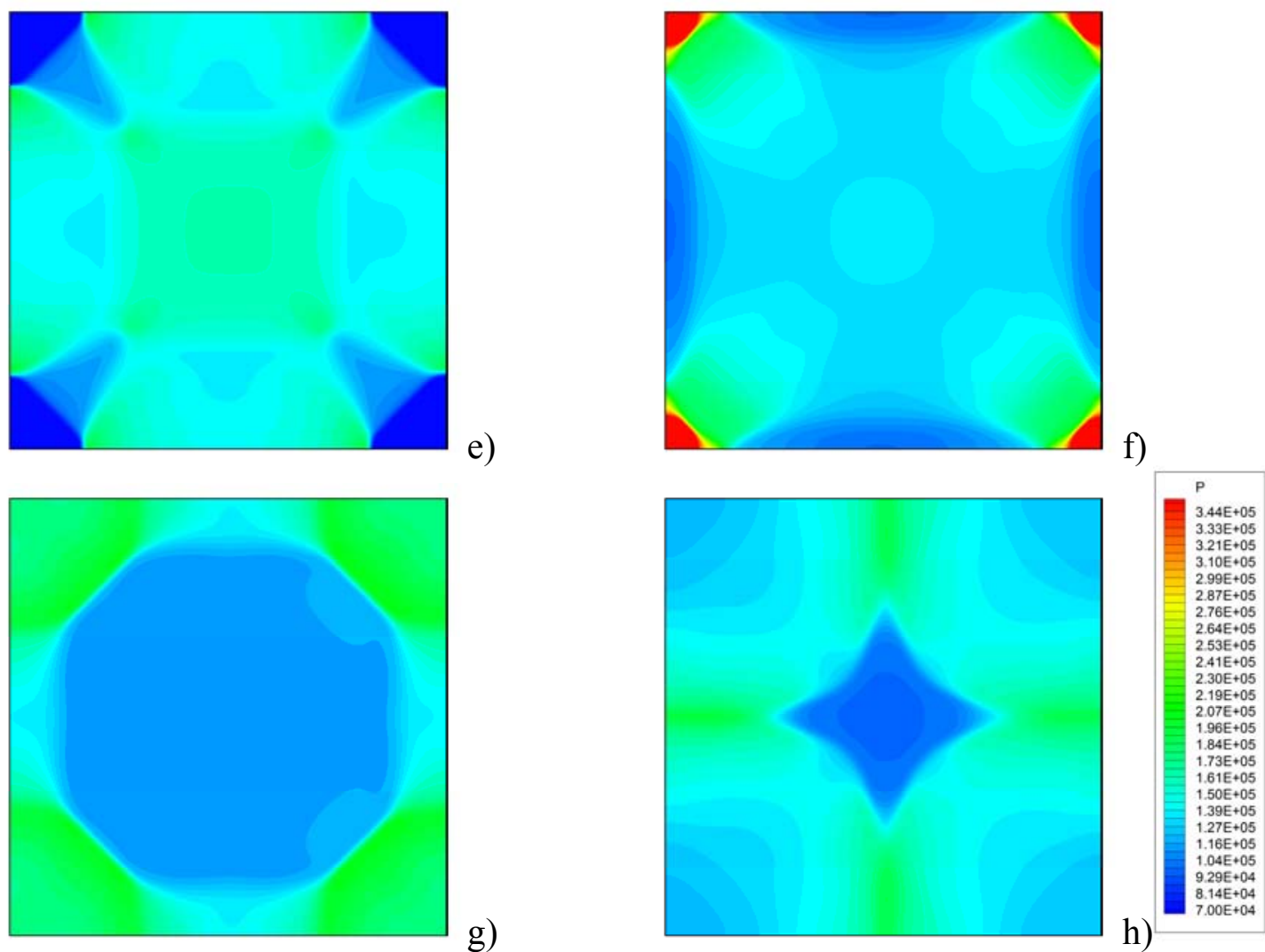

Figure 11: Snapshots of pressure distribution at eight evenly distributed times for $M_{s}=4.5$ and $\eta=0$ (no particles): $t_{\mathrm{o}}=1.25 \times 10^{-3} \mathrm{~s}, \delta t=5 \times 10^{-4} \mathrm{~s}$. 


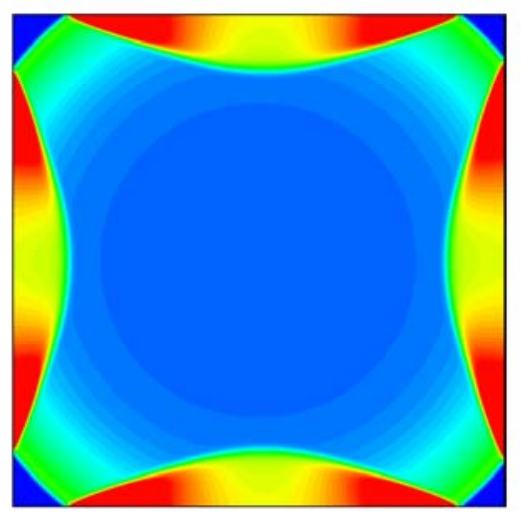

a)
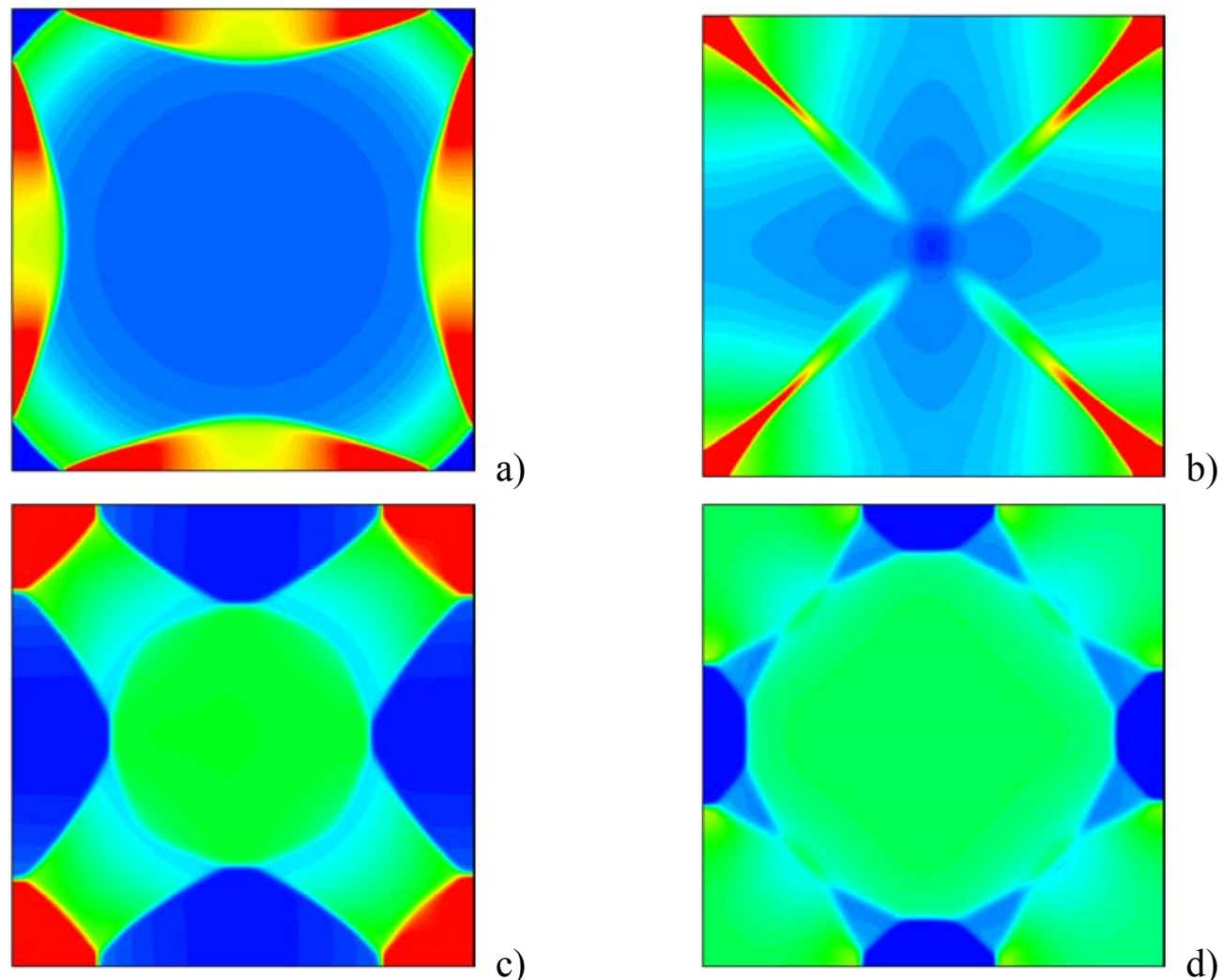

b)

c)

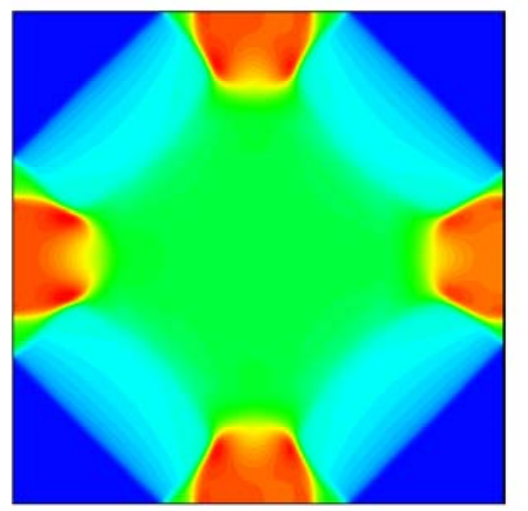

d)
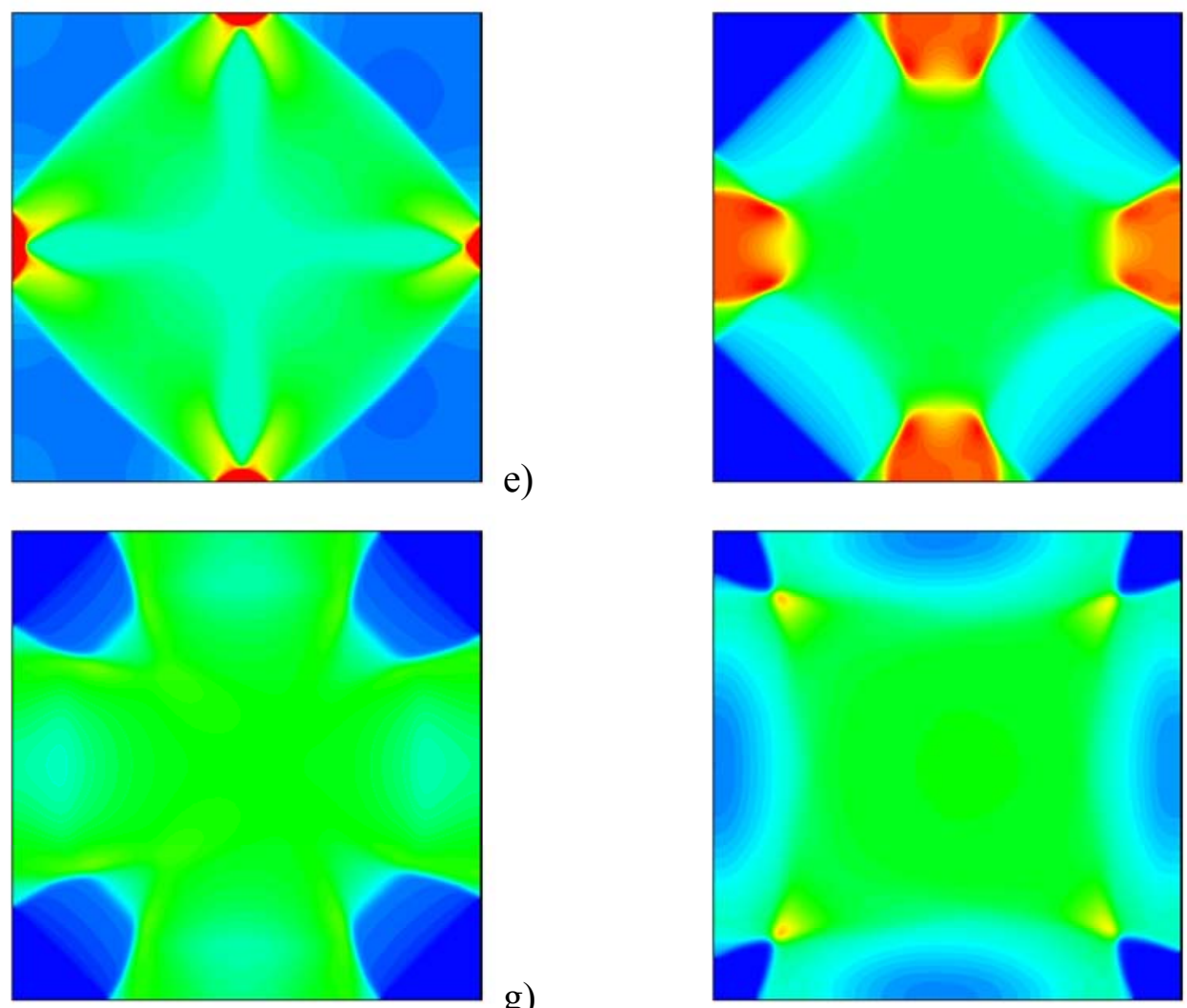

f)

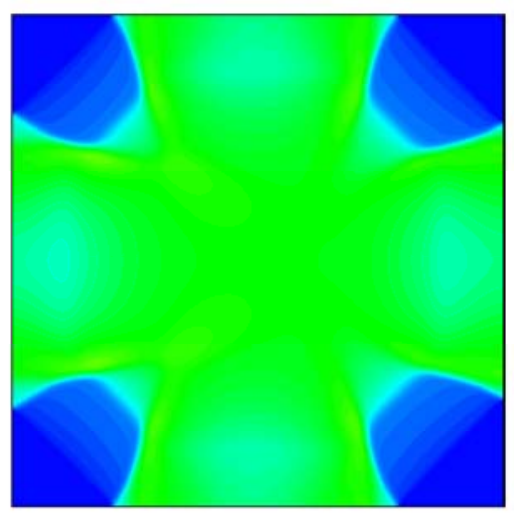

g)

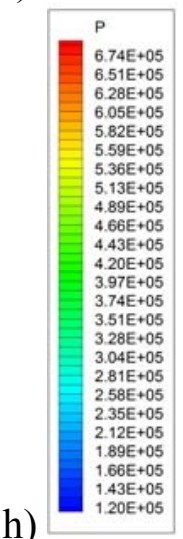

Figure 12: Snapshots of pressure distribution at eight evenly distributed times for $M_{s}=10$ and $\eta=0$ (no particles): $t_{\mathrm{o}}=5 \times 10^{-4} \mathrm{~s}, \delta t=2 \times 10^{-4} \mathrm{~s}$. 
The interplay among such asynchronous events leads to a typical flow topology displaying fascinating polygonal structures inside the cavity, with sides oriented alternately along the Cartesian or diagonal directions and with an increasing degree of complexity as time progresses.

Depending on the initial strength of the shock wave, this sequence of events can be repeated several times in practice to produce a rhythmic alternation in time of shock waves traveling toward the external boundary and then moving back toward the center, as shown in Figs. 11 and 12.

The addition of particles has dramatic consequences for the global thermofluid-dynamics. Related results are summarized in Figs. 13 and 14 for $M_{s}=4.5$ and 10, respectively (to be compared frame by frame with the equivalent contour maps at exactly the same instants in Figs. 11 and 12). These confirm that, by having an impact on the shock-wave propagation velocity, the particles interfere with all such phenomena, causing significant changes in the time evolution of the field depending on the specific values of $M_{s}, \eta$, and $R_{p}$ considered.

The related snapshots showing the effective distribution of particles as a function of time are shown in Figs. 15 and 16. As can be seen from these figures, the most striking feature of the particle dynamics in all cases is that the central region of the cavity tends to become depleted. As time progresses, the particles become increasingly clustered in a relatively narrow region adjacent to the solid boundaries. Interestingly, it can be seen that the particles remain there, forming a thick ring or "corona" surrounding a clear center. This corona behaves as a cohesive entity for a long time, as clearly witnessed by the existence of a sharp boundary between the particle-filled region and the clear fluid inside.

The key to understanding this scenario is the fact that, because of particle inertia, the fluid-particle momentum exchange by which the fluid is decelerated and the particles are accelerated is not an instantaneous process. Rather, the "feedback loop" is a somewhat gradual one that operates on a time scale comparable to that for the shock wave to travel from the center to the walls (or vice versa).

Particles typically "lag behind" the shock front. The irreversibility of particles that do not move toward the center when the shock-wave propagation is reversed is strongly correlated with the fact that, after all particles have been relocated to the periphery of the domain by the impact of the first outward-travelling shock wave (originating from the "ball" of initial conditions defined in Sect. IIG), their inertia prevents them from following the fast oscillations of the velocity field produced by fluid moving back along the radial direction. This mechanism depends sensitively on the density difference between the particles and the surrounding fluid, which in turn depends on the initial value of the shock Mach number (for both values of $M_{s}$ considered here, the response of particles to the large-scale flow produced by alternating shocks wave is strongly asynchronous).

As just explained, the temporal and spatial scales governing such dynamics are dependent on particle inertia and drag (i.e., density and size) and on properties (i.e., velocity) of the large-scale flow. However, there is another (even more important) factor for which particles tend to become trapped in the external corona. Wall-incident shock waves always propagate faster than reflected 
ones. This effect, together with the aforementioned intrinsic inertial effects, is responsible for the observed stable clustering of particles near the solid boundary.
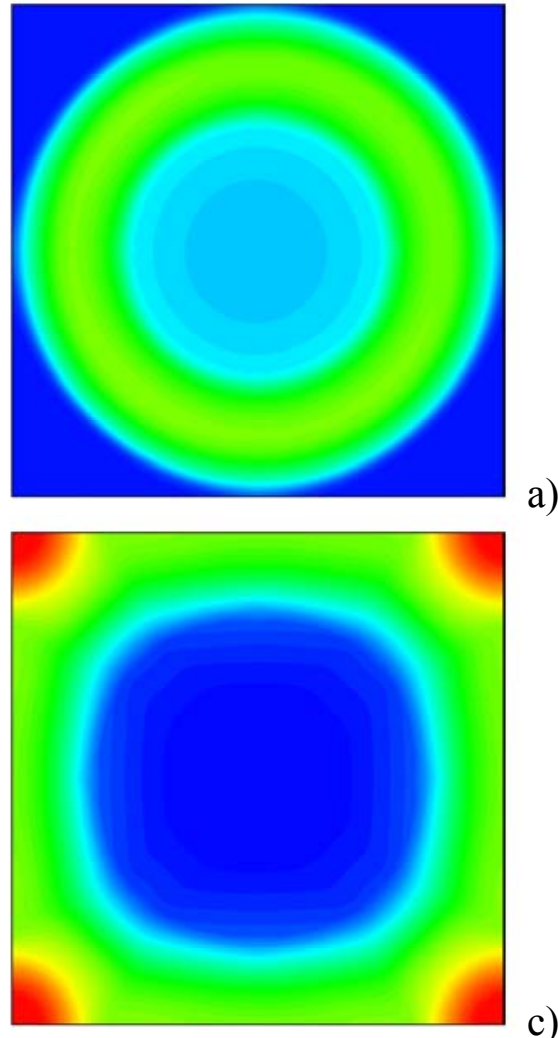

c)
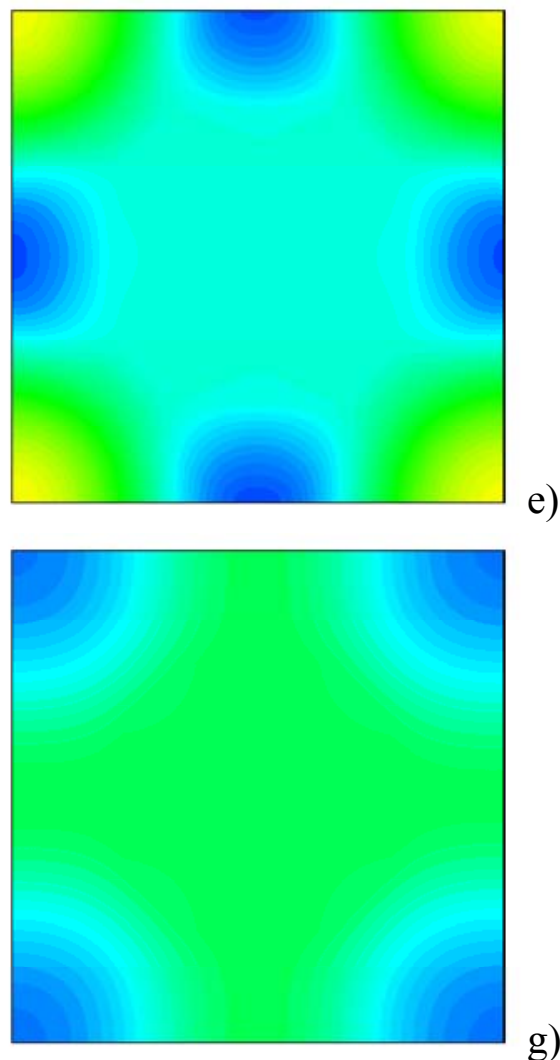

g)
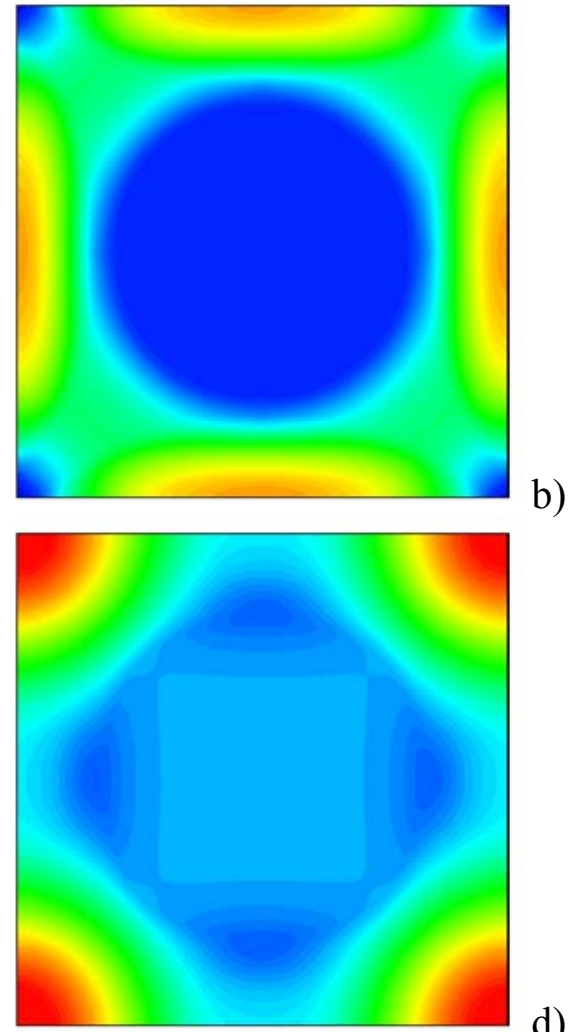

d)
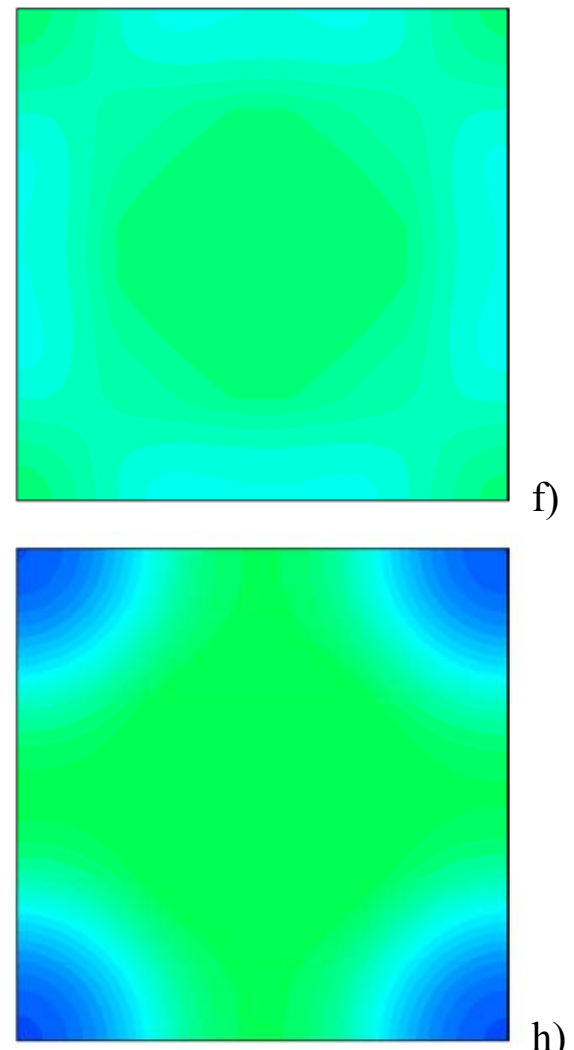

f)

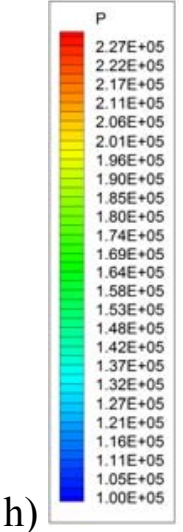

Figure 13: Snapshots of pressure distribution at eight evenly distributed times for $M_{s}=4.5$ and $\eta=2$ $\left(R_{p}=12.5 \mu \mathrm{m}\right): t_{0}=1.25 \times 10^{-3} \mathrm{~s}, \delta t=5 \times 10^{-4} \mathrm{~s}$. 

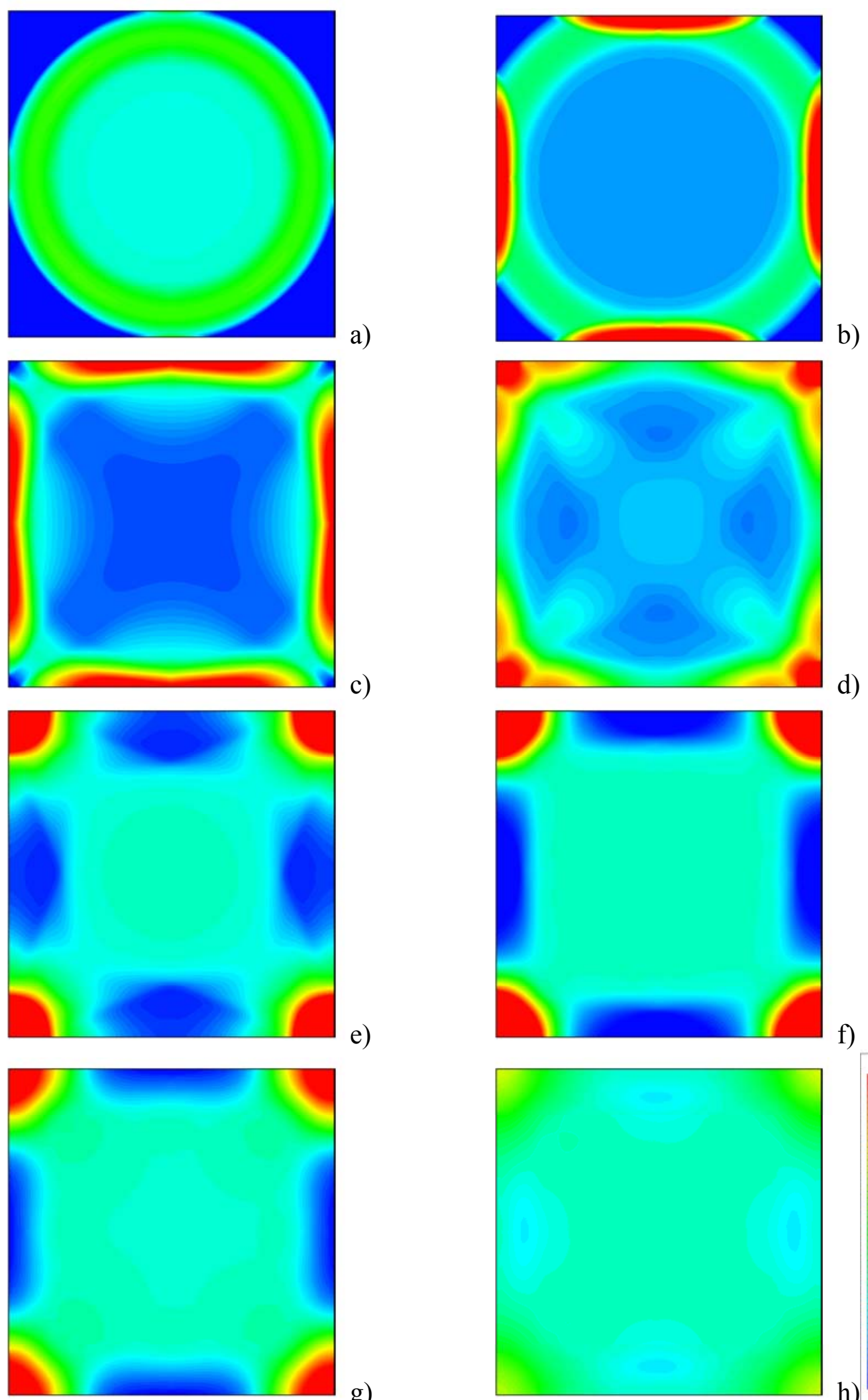

)

f)

g)

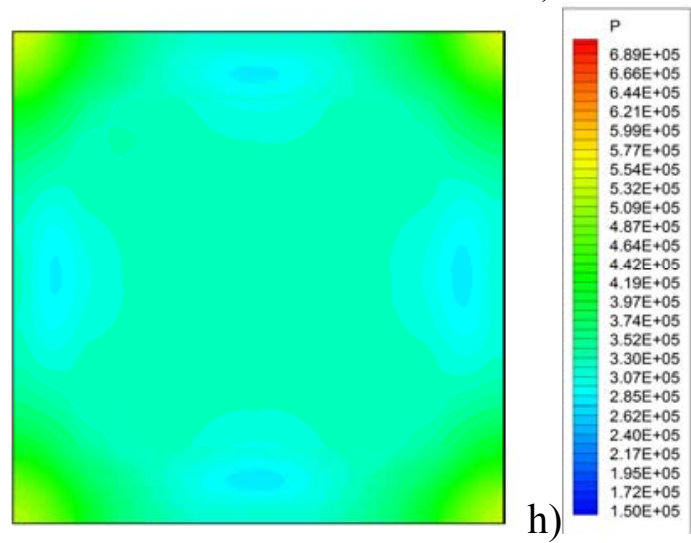

Figure 14: Snapshots of pressure distribution at eight evenly distributed times for $M_{s}=10$ and $\eta=2$ $\left(R_{p}=12.5 \mu \mathrm{m}\right): t_{\mathrm{o}}=5 \times 10^{-4} \mathrm{~s}, \delta t=2 \times 10^{-4} \mathrm{~s}$. 

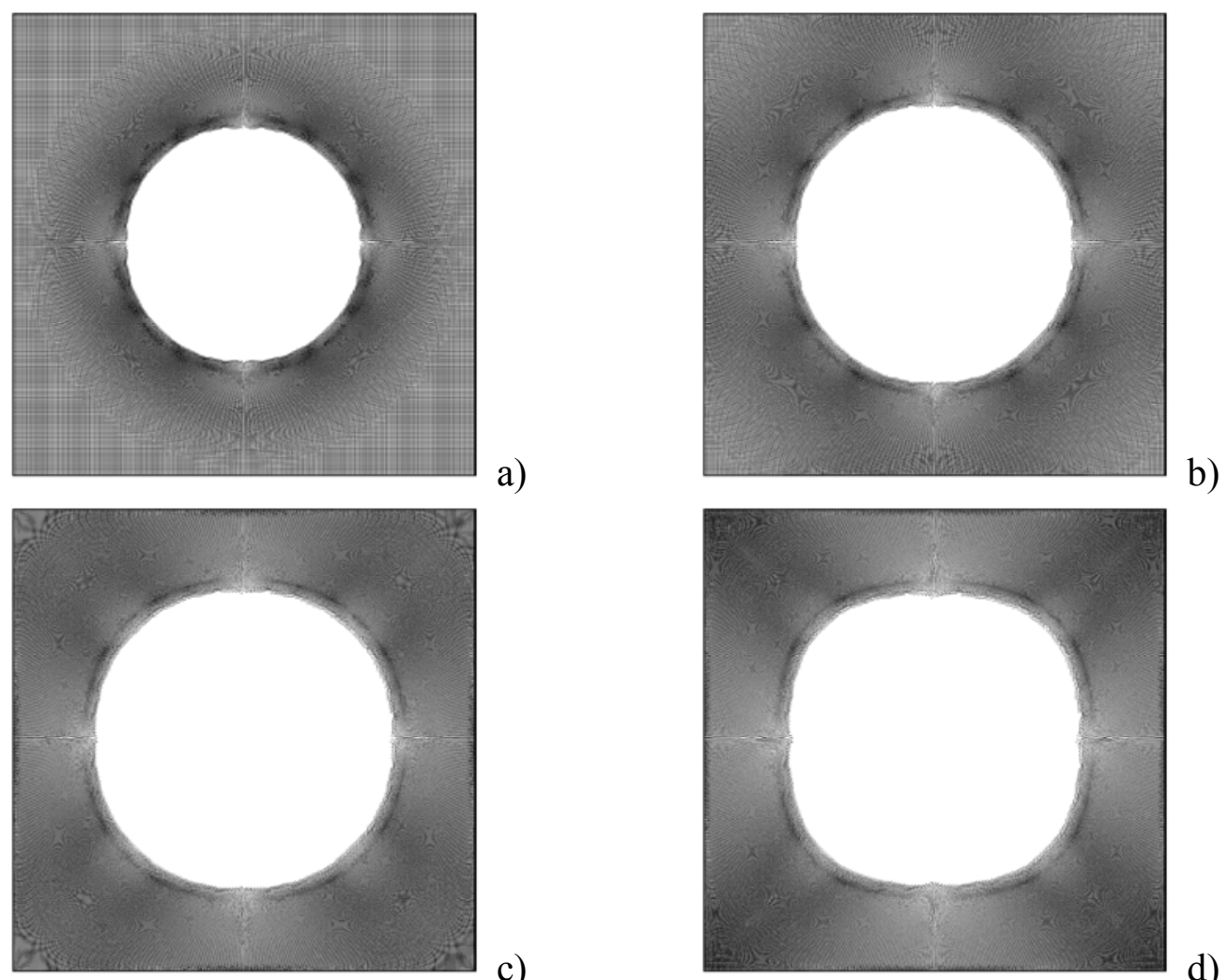

c)

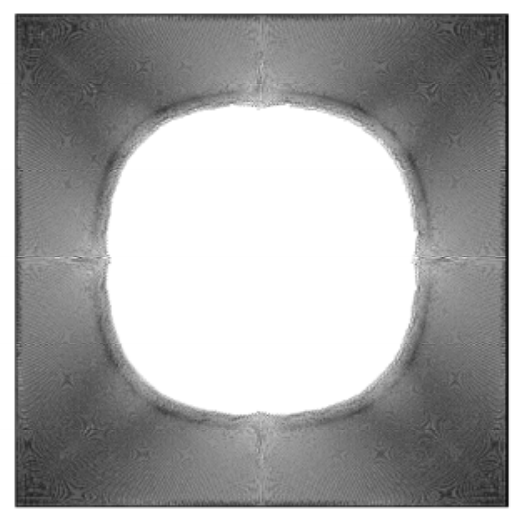

b)

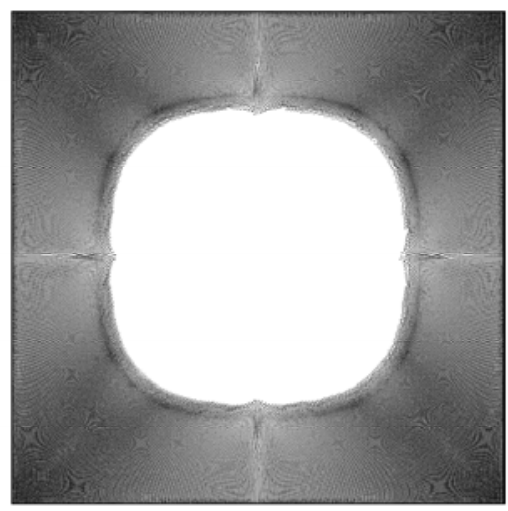

e)
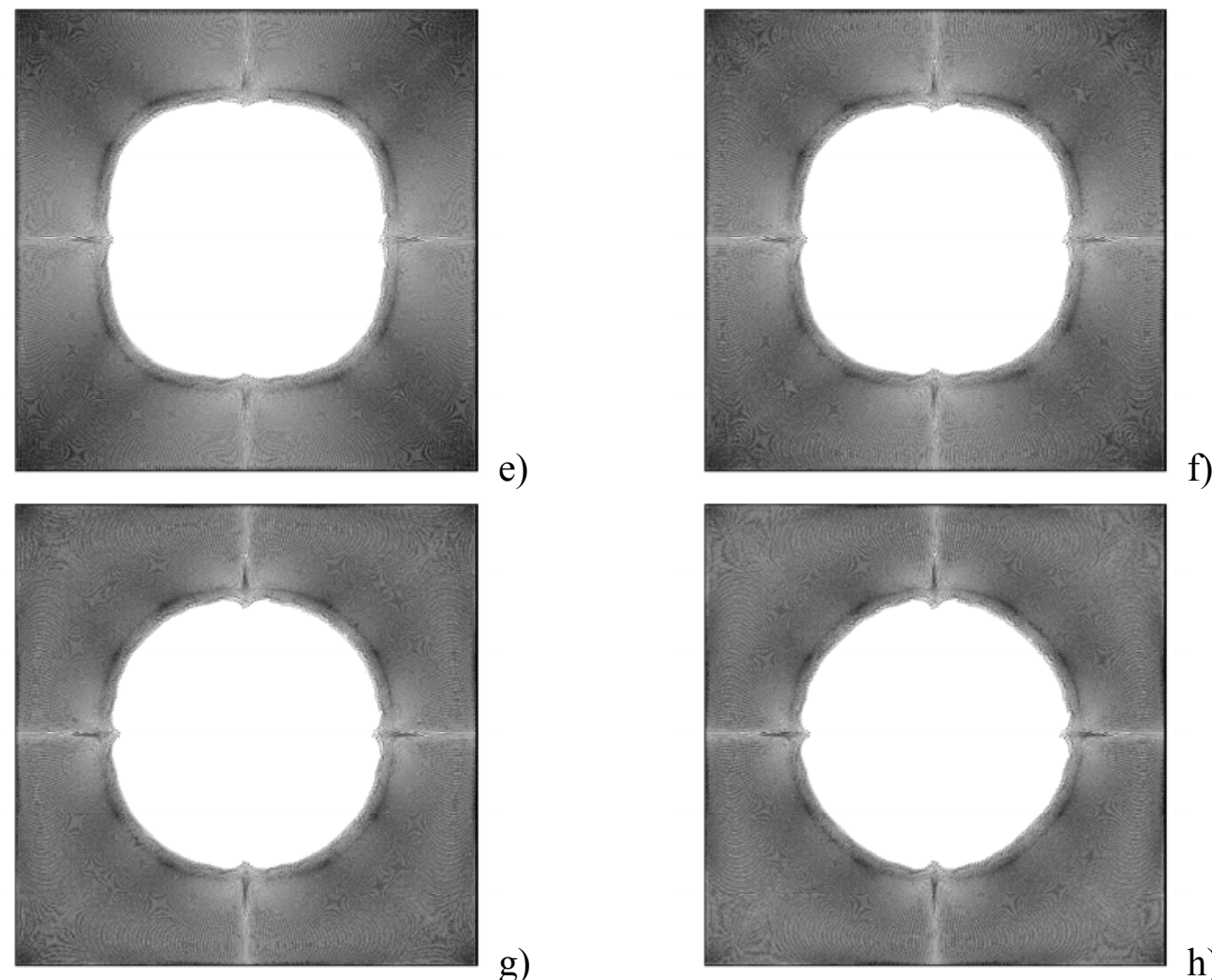

g)

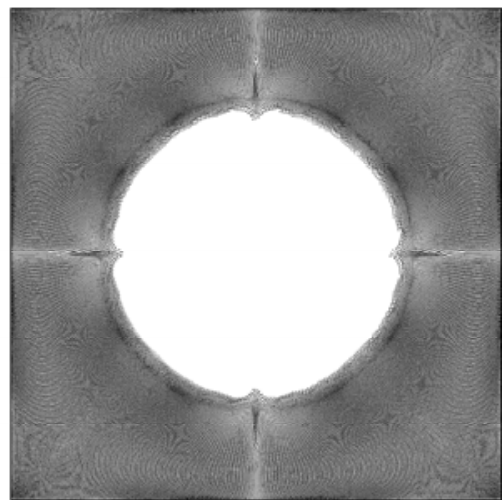

h)

Figure 15: Snapshots of particle distribution at eight evenly distributed times for $M_{S}=4.5$ and $\eta=2$ $\left(R_{p}=12.5 \mu \mathrm{m}\right): t_{0}=1.25 \times 10^{-3} \mathrm{~s}, \delta t=5 \times 10^{-4} \mathrm{~s}$. 

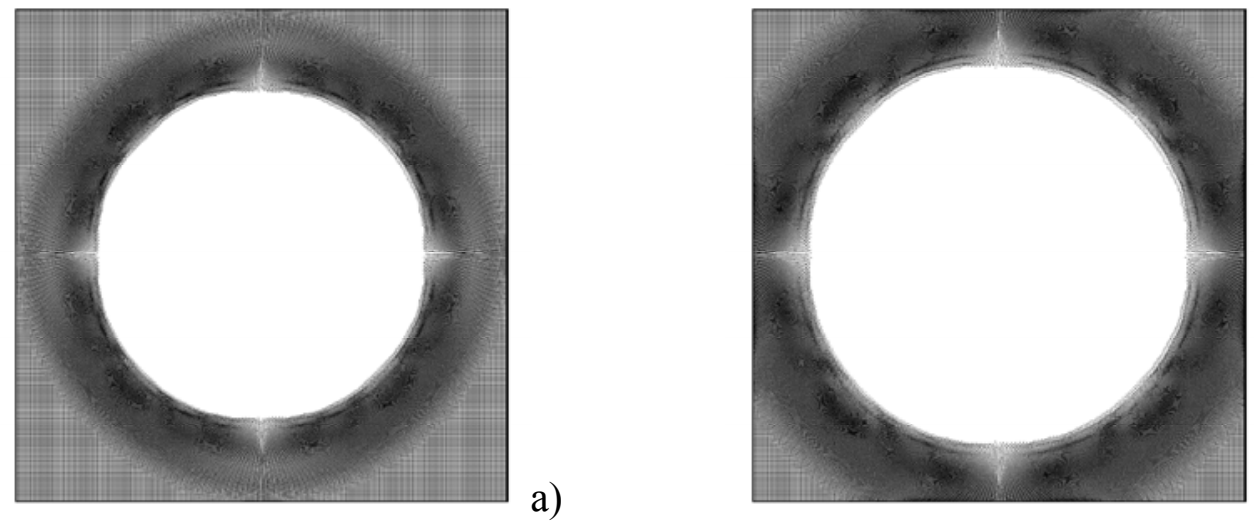

b)

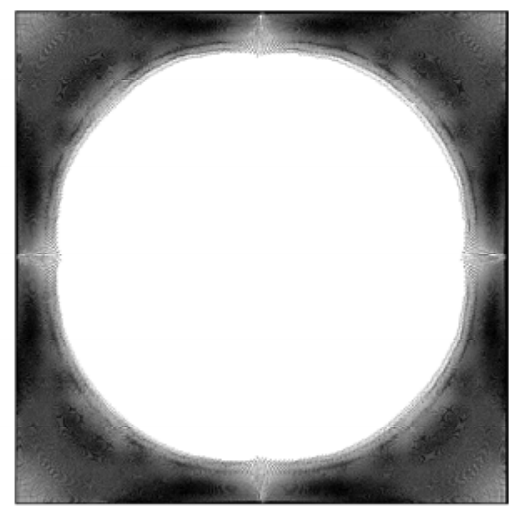

)

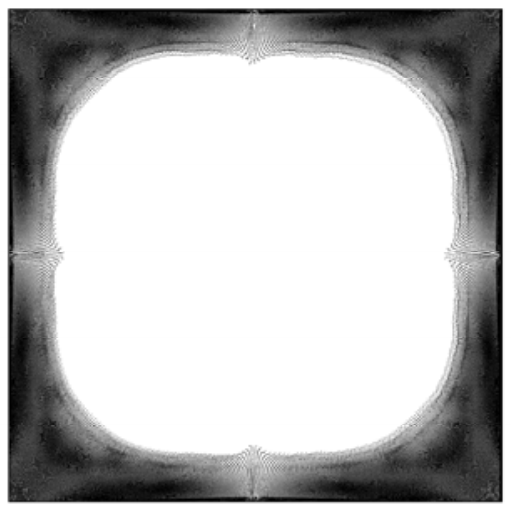

d)

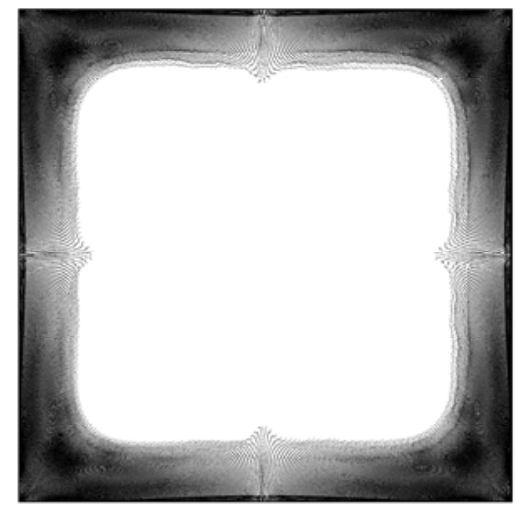

c)

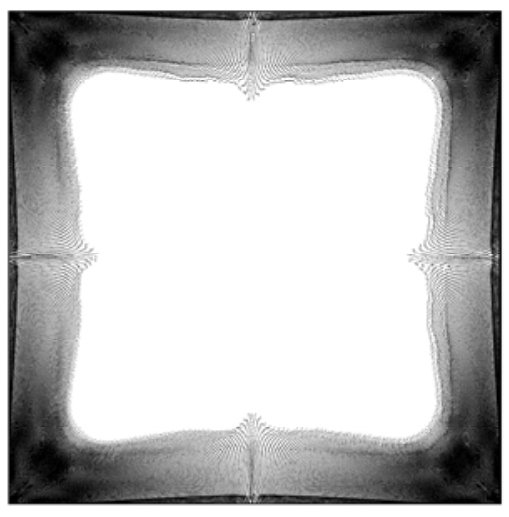

f)
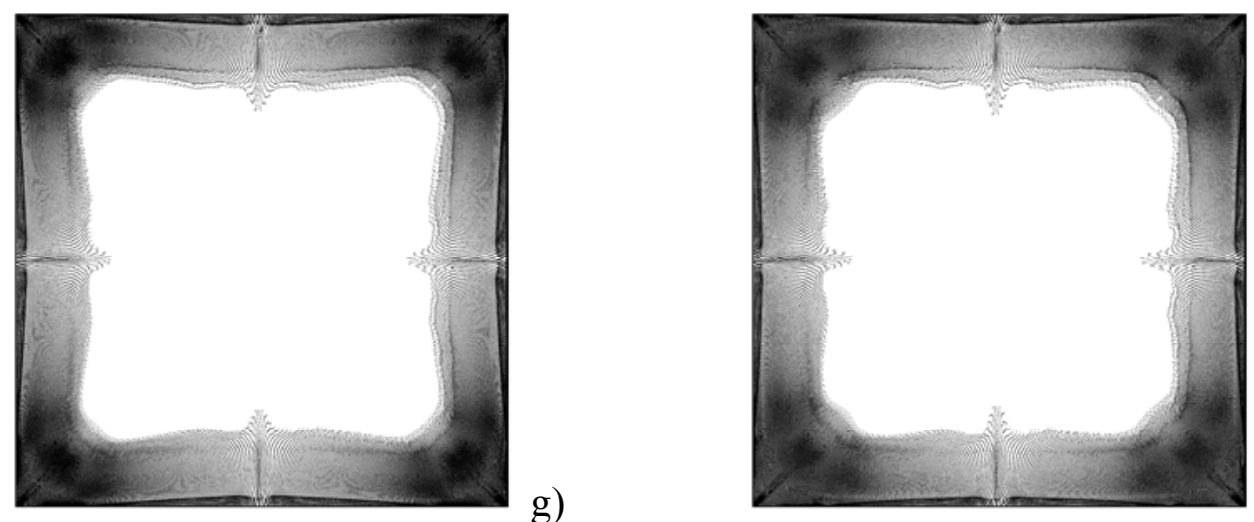

h)

Figure 16: Snapshots of particle distribution at eight evenly distributed times for $M_{s}=10$ and $\eta=2$ $\left(R_{p}=12.5 \mu \mathrm{m}\right): t_{\mathrm{o}}=5 \times 10^{-4} \mathrm{~s}, \delta t=2 \times 10^{-4} \mathrm{~s}$. 
Comparison of Figs. 15 and 16 for $M_{s}=4.5$ and 10, respectively, also reveals that, for fixed particle size, the radial extent of the corona of particles is larger for smaller values of the initial shock Mach number. This further confirms the interpretation just given about the dominant role played by inertial effects in determining such a scenario.

Interestingly, as time progresses, some "pulsations" can be seen in the radial position of the line separating the region containing particles from the pure liquid at the center (particle-depleted region). This can be regarded as a consequence of shock reflections, which still occur (albeit less frequently than for $\eta=0$ ) in the presence of dispersed solid mass. Although reflected shocks travelling inward cannot displace particles toward the center again, they lead to some weak (but noticeable) modulation of their position.

Moreover, for the larger value of $M_{s}$ considered, a "second front" becomes clearly visible in the distribution of particles. This appears as a second boundary separating the particles located in the corona region from a new, more "dense" (in terms of particle number) ring located further out. Accurate inspection of Fig. 16 (frames (d)-(h)) reveals that this second, apparently denser, ring results from particles bouncing off the solid wall and being reflected inward.

The influence exerted by the particle radius on the patterning behavior at a selected representative instant is shown in Figs. 17 and 18 for $\eta=2$ and $M_{s}=4.5$ and $M_{s}=10$, respectively.
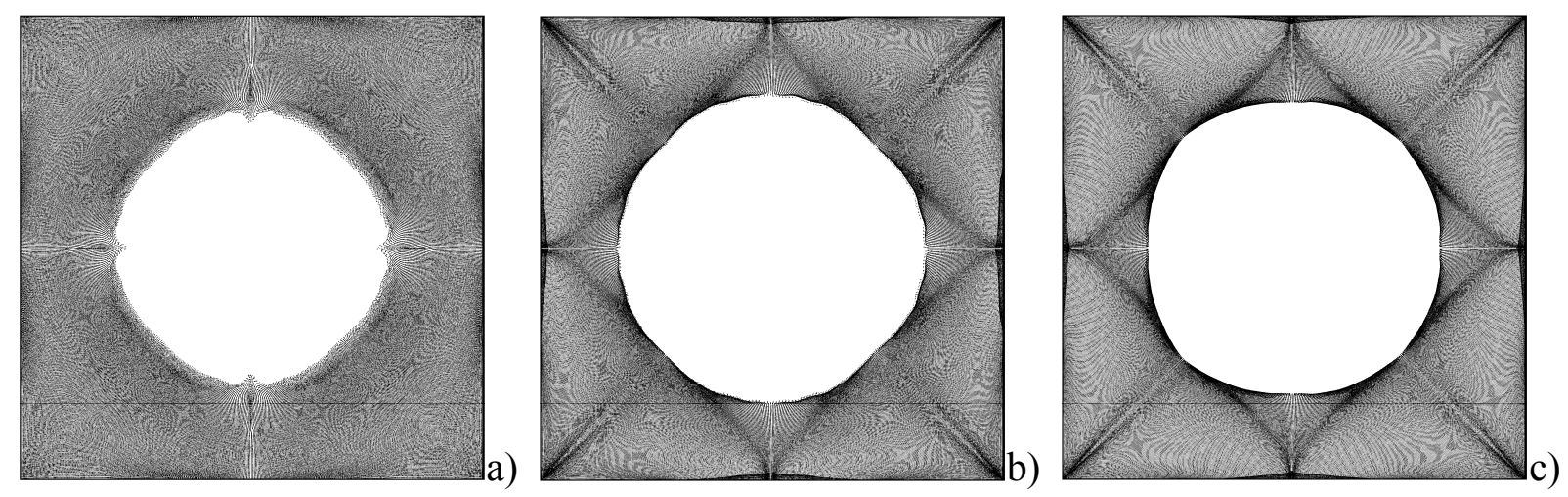

Figure 17: Snapshots of particle distribution at $t \cong 5 \times 10^{-3}$ for $M_{s}=4.5, \eta=2$ and different values of the particle radius: a) $R_{p}=12.5 \mu \mathrm{m}$ (area covered by particles $\left.\left.\mathrm{A}_{\mathrm{p}} \cong 0.74\right), \mathrm{b}\right) R_{p}=25 \mu \mathrm{m}\left(\mathrm{A}_{\mathrm{p}} \cong 0.66\right)$, c) $R_{p}=50 \mu \mathrm{m}\left(\mathrm{A}_{\mathrm{p}} \cong 0.67\right)$. 

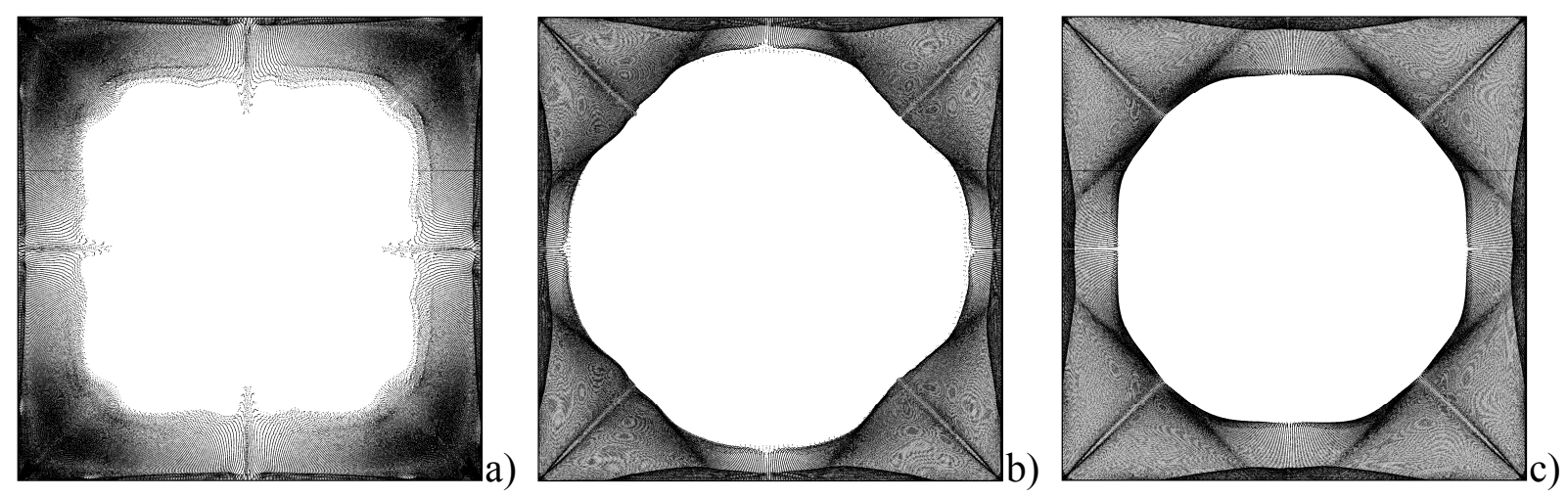

Figure 18: Snapshots of particle distribution at $t \cong 2 \times 10^{-3}$ [s] for $M_{S}=10, \eta=2$ and different values of the particle radius: a) $R_{p}=12.5 \mu \mathrm{m}$ (area covered by particles $\left.\mathrm{A}_{\mathrm{p}} \cong 0.5\right)$, b) $R_{p}=25 \mu \mathrm{m}\left(\mathrm{A}_{\mathrm{p}} \cong 0.42\right)$, c) $R_{p}=50 \mu \mathrm{m}\left(\mathrm{A}_{\mathrm{p}} \cong 0.50\right)$.

Such figures indicate that, in general, an increase in the particle size with respect to the smallest considered radius (namely $R_{p}=12.5 \mu \mathrm{m}$ ) results in a shrinkage of the area of the portion of the physical domain occupied by particles (i.e. the area of the "corona", hereafter denoted by $\mathrm{A}_{\mathrm{p}}$ ). This effect can be made very evident by comparing directly the results for $R_{p}=12.5 \mu \mathrm{m}$ with those for $R_{p}=25 \mu \mathrm{m}$. As an example, for $M_{s}=4.5$ the reduction experienced by $\mathrm{A}_{\mathrm{p}}$ by doubling the value of $R_{p}$ is $\cong 11 \%$ (Fig. 17). Notably, an increase in the initial shock Mach number from 4.5 to 10 , raises such a percentage to $16 \%$ (Fig. 18). Obviously, such effects have to be ascribed once again to the influence exerted by the dispersed solid matter on the outward traveling shock waves (which tend to push the particles towards the solid boundary). As shown in Figs. 5-10 and repeatedly mentioned in the preceding text, for a fixed value of $\eta$, the smaller the particle radius, the stronger the mitigating role particles play on the velocity of propagation and strength of the shock waves.

When the pulsations (with shock waves moving back and forth along the radial direction) finally end (because the system has reached a condition of almost uniform pressure inside the cavity), some interesting "geometrical" shapes can be identified in the particle distribution. Such shapes range from the octagonal pattern visible in Fig. $19 \mathrm{~b}$ to the aesthetically appealing "cloverleaf" in Fig. 19d, passing through the intermediate stage of "tetraconch" of four-shell shape evident in Fig. 19c. Such beautiful patterns can be regarded as an outcome (or "tangible manifestation") of the complex interplay established over time among different mechanisms: the propagation of shock waves, their reflection dynamics, and the ability of the resulting time-dependent flow to transport inertial particles according to their mass and specific size (the presence of particles, in turn, exerting a back influence of the properties of the emerging macroscopic flow). 

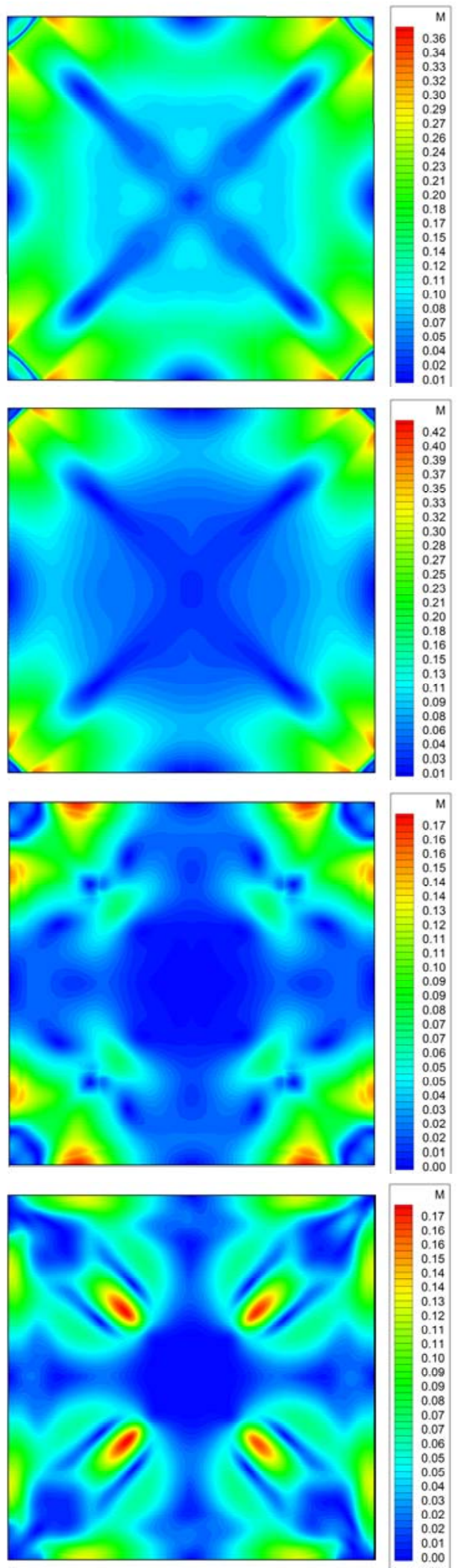

a)

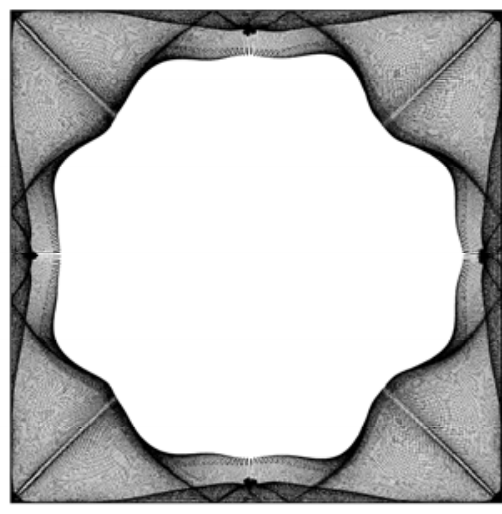

b)

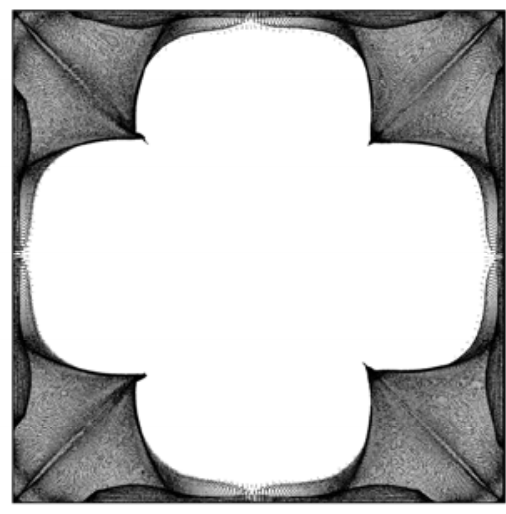

c)

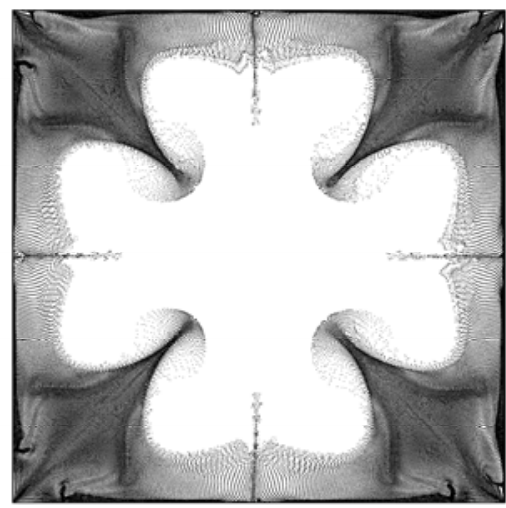

d)

Figure 19: Snapshots of Mach number (left) and particle distribution (right) at $t=4 \times 10^{-3}[\mathrm{~s}]$ for $M_{s}=10, \eta=2$ and different values of the particle radius: a) $R_{p}=0 \mu \mathrm{m}$ (no particles), b) $R_{p}=50 \mu \mathrm{m}, \mathrm{c}$ ) $\left.R_{p}=25 \mu \mathrm{m}, \mathrm{d}\right) R_{p}=12.5 \mu \mathrm{m}$, 


\section{Conclusions}

A specific mathematical and numerical framework has been developed to determine the effects of dispersed solid particles on the propagation of a shock wave inside a closed cavity. A rich spectrum of convective dynamics has been revealed, consisting of multiple reflections that lead to interesting patterns and particulate structures inside the cavity. Such phenomena are governed by different coexisting mechanisms, including flow compressibility, shock-wave formation, particle inertia, and "viscous effects" at small length scales. The related interconnected feedback loops and "iterative" process make the system extremely sensitive to the (albeit relatively small) internal variations produced by changes in the shock propagation velocity or the particle properties.

The numerical simulations reveal that the dynamics for particles with large diameters or small mass loadings are not appreciable different from those of a pure gas. However, increasing the total surface area of the particles tends to enhance the ability of the dispersed mass to mitigate the effects of the detonation. Such an increase in total area can be achieved by reducing the typical particle size for a given mass load and/or by increasing the overall particle number for a fixed particle size.

Moreover, particles attenuate shock waves more rapidly for larger mass fractions. By slowing down the shock, particles also exert a profound influence on the sequence of evolutionary stages displayed by the system (causing a significant decrease in the number of shock reflections over a given observation timeframe). By weakening the shock wave significantly, the particles also have a remarkable impact on the instantaneous wall pressure distributions.

The initial strength of the shock wave, obviously, also plays a significant role in such dynamics. Indeed, an increase in $M_{s}$ has two distinct outcomes: it determines an amplification of the damping effect (in percentage) exerted by particles on the pressure load for a fixed mass load, and it causes a shrinkage of the radial extension of the "corona" where particles tend to cluster (this accumulation region behaves as a cohesive entity for relatively long times).

Although our numerical framework allowed us to explore most (but not all) of the features and quantities of interest, further elaboration of the model may be required in the future, in particular to account for other effects eventually necessary for an even more accurate quantitative description. Among these, we cite the additional forces recently identified by Parmar et al. ${ }^{34}$ or the particlesfluid heat-exchange process. The former depend on time-history integrals that would significantly increase the complexity of the mathematical model, while the latter would require the consideration of an additional Lagrangian transport equation and additional coupling terms in the Eulerian energy equation. Future work could also be devoted to account for the molecular vibrational degree of freedom that is excited above a given temperature threshold, this having remarkable consequences for gaseous thermodynamic properties such as viscosity and specific heat coefficients. Moreover, though we did not detect any fluid-dynamic instability for the present conditions, we might further expand the ranges of considered parameters and/or investigate the case of initially non-symmetric (“eccentric") detonations. 


\section{References}

[1] Thornber B., Drikakis D., Youngs D. L. and Williams R. J. R., (2010), The influence of initial conditions on turbulent mixing due to Richtmyer-Meshkov instability, J. Fluid Mech., 654: 99-139. [2] Lappa M., (2016), On the nature, formation and diversity of particulate coherent structures in Microgravity Conditions and their relevance to materials science and problems of Astrophysical interest, Geophysical and Astrophysical Fluid Dynamics, 110(4): 348-386.

[3] Sivier S., Loth E., Baum J., Löhner R., (1994), Unstructured adaptive remeshing finite element method for dusty shock flow, Shock Waves, 4:15-23.

[4] Boiko V.M., Kiselev V.P., Kiselev S.P., Papyrin A.N., Poplavskil S.V., and Fomin V.M., (1996), Interaction of a shock wave with a cloud of particles, Combustion, Explosion, and Shock Waves, 32, (2): 191-203.

[5] Chang E.J. and Kailasanath K., (2003), Shock wave interactions with particles and liquid fuel droplets, Shock Waves, 12: 333-341.

[6] Sommerfeld M., (1985), The unsteadiness of shock waves propagating through gas-particle mixtures, Experiments in Fluids, 3:197-206.

[7] Shepherd J. E., (2009), Structural Response of Piping to Internal Gas Detonation, Journal of Pressure Vessel Technology,131:031204 (13 pages).

[8] Dhakal R.P., (2004), Explosion induced structural response: An overview, NZSEE Conference 2004.

[9] Chojnicki K., Clarke A.B., and Phillips J.C., (2006), A shock-tube investigation of the dynamics of gas-particle mixtures: Implications for explosive volcanic eruptions, Geophysical Research Letters, 33: L15309 (5 pages).

[10] de' Michieli Vitturi M., Esposti Ongaro T., Neri A., Salvetti M.V., Beux F., (2007), An immersed boundary method for compressible multiphase flows: application to the dynamics of pyroclastic density currents, Comput. Geosci., 11:183-198.

[11] Erfani R., Zare-Behtash H., and Kontis K. (2012), Influence of shock wave propagation on dielectric barrier discharge plasma actuator performance, Journal of Physics D: Applied Physics, 45(22), 225201.

[12] Ukai T., Zare-Behtash H., Kontis K., and Obayashi S. (2016), Three-dimensional shock wave distortion in shock-square vortex loop interaction. Experimental Thermal and Fluid Science, 79: 8590.

[13] Rudinger G. and Chang A., (1964), Analysis of nonsteady two phaseflow, Phys. Fluids 7: 1747-1754.

[14] Marconi F., Rudman S., Calia V., (1981), Numerical study of one-dimensional unsteady particle-laden flows with shock, AIAA Journal. 19: 1294-1301.

[15] Higashino F., (1983), Characteristics method applied to blast waves in a dusty gas, $Z$. Naturforsch. 38a: 399-406.

[16] Carrier G.F., (1958), Shock waves in a dusty gas, J. Fluid Mech., 4: 376-382.

[17] Soo S.L., (1961), Gas-dynamic processes involving suspended solids, AIChE J., 7(3): 384-391.

[18] Kriebel A.R., (1964), Analysis of normal shock waves in particle-laden gas, J. Basic. Eng., 86(4): 655-665.

[19] Rudinger G., (1964), Some Properties of Shock Relaxation in Gas Flows Carrying Solid Particles, Phys. Fluids, 7(5): 658-663. 
[20] Olim M., Ben-Dor G., Mond M., and Ingra O., (1990), A general attenuation law of moderate planar shock waves propagating into dusty gases with relatively high loading ratios of solid particles, Fluid Dynamics Research, 6(3-4): 185-200.

[21] Aizik F., Ben-Dor G., Elperin T., Igra O., Mond M., (1995), Attenuation Law of Planar Shock Waves Propagating Through Dust-Gas Suspensions, AIAA journal, 3( 5): 953-955.

[22] Loth E., Sivier S. and Baum J., (1997), Dusty Detonation Simulations with Adaptive Unstructured Finite Elements, AIAA journal, 35(6): 1018-1024.

[23] Marconi F., (1994), Investigation of the interaction of a blast wave with an internal structure, AIAA journal, 32(8): 1561-1567.

[24] Bagabir A. M. and Drikakis D., (2005), Shock wave induced instability in internal explosion, (2005), Aeronautical Journal -New Series- 109:1101 (36 pages).

[25] Crowe C.T., (1982), Review numerical models for dilute gas-particle flows, J. Fluid Eng., 104:297-303.

[26] Marble F. E., (1970), Dynamics of dusty gases, Ann. Rev. Fluid Mech., 2: 397-446.

[27] Clift R, Grace J.R, Weber M.E., (1978), Bubbles, Drops, and Particles, Academic Press, New York.

[28] Lappa M., (2013), On the variety of particle accumulation structures under the effect of gjitters, J. Fluid Mech., 726: 160-195.

[29] Hirsch C., (2002), Numerical computation of internal and external flows: volume 2: computational methods for inviscid and viscous flows. Chichester, John Wiley \& Sons, 2002.

[30] Laney C.B., (1998), Computational Gas-dynamics, Cambridge University Press.

[31] Anderson J., (1995), Computational fluid dynamics: the basics with applications, Mcgraw Hill, New York, 1995.

[32] Sakurai A., (1965), Blast wave theory, Basic developments in Fluid Dynamics, edited by M. Holts, Vol. 1, Academic Press, New York, 1965, pp. 309-375.

[33] Chebotareva E.I., Aleshin A.N., Zaytsev S.G., (1999), Investigation of interaction between reflected shocks and growing perturbation on an interface, Shock Waves, 9: 81-86.

[34] Parmar M., Haselbacher A. and Balachandar S., (2012), Equation of motion for a sphere in non-uniform compressible flows, J. Fluid Mech., 699, 352-375. 